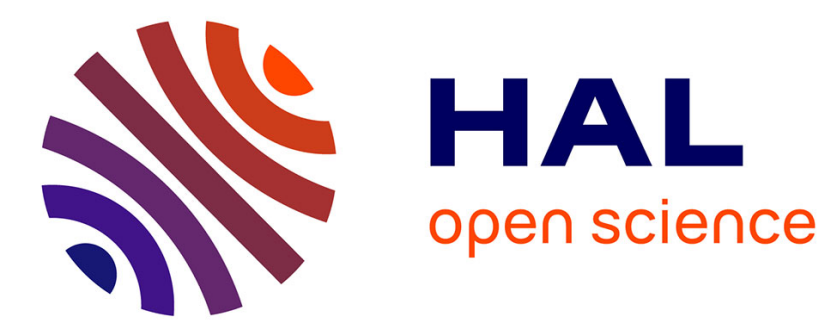

\title{
Near-wall aerodynamic response of an acoustic liner to harmonic excitation with grazing flow
}

Olivier Léon, Fabien Méry, Estelle Piot, Claudia Conte

\section{To cite this version:}

Olivier Léon, Fabien Méry, Estelle Piot, Claudia Conte. Near-wall aerodynamic response of an acoustic liner to harmonic excitation with grazing flow. 2019. hal-02087873

\section{HAL Id: hal-02087873 https://hal.science/hal-02087873}

Preprint submitted on 2 Apr 2019

HAL is a multi-disciplinary open access archive for the deposit and dissemination of scientific research documents, whether they are published or not. The documents may come from teaching and research institutions in France or abroad, or from public or private research centers.
L'archive ouverte pluridisciplinaire HAL, est destinée au dépôt et à la diffusion de documents scientifiques de niveau recherche, publiés ou non, émanant des établissements d'enseignement et de recherche français ou étrangers, des laboratoires publics ou privés. 


\title{
Near-wall aerodynamic response of an acoustic liner to harmonic excitation with grazing flow
}

\author{
Olivier Léon, Fabien Méry, Estelle Piot, Claudia Conte
}

April 2, 2019

\begin{abstract}
The near-orifice aerodynamic response of a single degree of freedom acoustic liner to tonal and multi-tonal excitation with grazing flow was experimentally studied. A high-magnification PIV setup was designed in order to provide dense $2 \mathrm{D}$ velocity field measurements above a millimeter-sized orifice of the liner. The resonator near-orifice velocity dynamics near and far from resonance were shown to be significantly different, with dynamic velocity scales well captured by a lumped-element model that was also satisfactorily applied to multi-tonal forcing cases. The effects of varying the forcing acoustic sound pressure level and the tangential flow velocity scale (the friction velocity) were investigated. It was observed that a "rough-wall" analogy was not suited to account for the induced aerodynamic effects, but that, under certain conditions, a "transpiration wall" analogy may be adequate.
\end{abstract}

Keywords High-Magnification PIV · Acoustic liner · Acoustic resonator dynamics

\section{Introduction}

Acoustic liners are largely used in the aeronautical industry to reduce the environmental noise impact of aircraft during take-off and landing. Such liners are usually installed within nacelles and rear ducts of turbofan engines for fan noise mitigation, or on external surfaces for airframe noise reduction. As illustrated in Fig. 1, conventional acoustic liners are made of honeycomb cavities bonded to a perforated face-sheet and closed by a rigid back-plate. This layout locally forms a set of acoustic resonators, that are cavities with small openings, whose geometry can be designed to match noise

ONERA, 2 avenue Edouard Belin, 31055 Toulouse, France E-mail: olivier.leon@onera.fr E-mail: fabien.mery@onera.fr

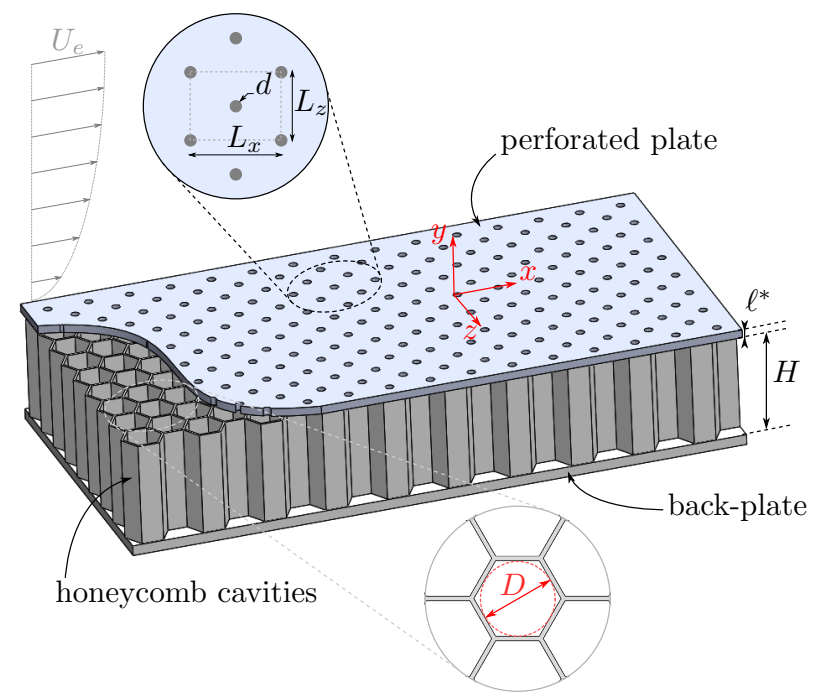

Fig. 1 Schematic of an acoustic liner composed of honeycomb cavities and topped with a perforated plate.

absorption requirements on a specified frequency bandwidth (Motsinger and Kraft 1991).

For aeronautical applications, a long-wavelength approximation is usually considered (i.e. the dimensions of the resonator are small compared to the wavelength of the acoustic waves to be attenuated, $H \ll \lambda_{a}$ ) and the resonator may be described using a (weakly non-linear) mass-spring-damper model (Ingard 1953; Rienstra and Singh 2018): upon external acoustic forcing, the mass of air in the opening (referred to as the "neck") moves, applying a load on the compressible air in the cavity that acts as a spring. At and near the resonance frequency, acoustic energy is then mainly dissipated by viscosity in the neck and by vortex shedding on both sides of the perforated face-sheet (Zhang and Bodony 2016).

An acoustic liner is commonly characterized by a normalized complex impedance $Z(\omega) \equiv \hat{p}(\omega) /\left(\rho_{0} c_{0} \hat{v}(\omega)\right)$ that can be seen as macroscopic parameter conveniently 
describing the liner response to an external acoustic forcing, as a function of the angular frequency $\omega$. It provides a simple boundary condition in the frequency domain that relates (in terms of amplitude and phase difference) spatially-averaged acoustic pressure fluctuations $\hat{p}$ and normal velocity fluctuations $\hat{v}$ at the wall. The real part of $Z$ is termed the resistance $R$ and the imaginary part is referred to as the reactance $X$.

This dimensionless impedance can be related to an absorption coefficient $\alpha(\omega)$ that measures the ratio of acoustic energy absorbed (that is dissipated or transferred to turbulence) by the liner to the energy of a normal incident acoustic wave, such that

$\alpha(\omega)=1-\left|\frac{1-Z}{1+Z}\right|^{2}$.

The aerodynamic (steady-state) response of such acoustic resonators is characterized by alternating inflow and outflow phases at its openings, with amplitudes that depend on the excitation. With no grazing flow and for low forcing levels, typically such that the induced fluid particle displacement $s$ in the opening is smaller than the neck length $\ell^{*}$ (Ingård and Labate 1950) and than the opening diameter $d$ (Rienstra and Hirschberg 2018), no significant flow separation around the orifice is observed and acoustic damping is mainly driven by viscous effects inside and near the opening. This regime can then be modeled in a linear framework (Melling 1973), such that $Z$ (and thus $\alpha$ ) does not significantly depend on the incident acoustic wave. It is well established, however, that increased sound intensities (Sivian 1935; Guess 1975) up to levels of practical interest for aeronautic applications or the addition of a grazing flow (Kirby and Cummings 1998) generally lead to non-linear effects. In this regime, the impedance $Z$ depends on both the intensity of the incident acoustic wave and on the characteristic velocity scale of the grazing flow (typically $U_{\tau}$, the mean friction velocity), implying that it cannot be considered as a wall property only.

These non-linear effects are mainly of hydrodynamic nature, with losses induced by circulation and turbulence promotion (Cummings 1984). Putting aside grazing flow effects, large sound pressure levels (SPL) induce inflow and outflow phases of significant amplitude, ultimately yielding "synthetic" jets issued on both sides of the resonator neck at the acoustic forcing frequency. The associated vortex shedding induces an increase in the resistance term $R$ of the resonator, that may yield an increase in the absorption coefficient $\alpha$ when $R$ is initially lower than 1, as illustrated in this work in Fig. 3. Of particular significance, experimental evidence supporting this picture has been reported by Ingård and
Labate (1950) for an orifice of varying geometry placed in a tube: near-orifice streamlines were then visualized using smoke illuminated by a light sheet. Furthermore, Ingard and Ising (1967) relied on hot-wire anemometry to measure the velocity fluctuations at the orifice of a single resonator with and without a bias flow, showing a direct link between $R$ and the measured velocity amplitude for large SPL. More recently, Roche et al (2009) and Zhang and Bodony (2012) performed Direct Numerical Simulations (DNS) on an isolated resonator with large SPL forcing, the latter authors suggesting an important role played by the orifice boundary layers in the non-linearity observed.

The addition of a grazing flow on the resonator also generally leads to an increase of the orifice resistance (Melling 1973). The physical explanation commonly provided is based on experiments such as the ones performed by Baumeister and Rice (1975) and Rogers and Hersh (1976) on an isolated resonator in a water tunnel: visualization of the streamlines suggests the formation of a recirculation region at the upstream edge of the orifice due to flow separation during the inflow phase. This separation yields what is called a vena contracta that reduces the effective open area of the resonator orifice. For the outflow phase, this vena contracta effect was observed to be less pronounced. Charwat and Walker (1983) performed pointwise velocity measurements inside and above a $2 \mathrm{D}$ slot Helmholtz resonator using a hot-film anemometer to study its aerodynamic response, highlighting such grazing flow effects. Following these experimental results, quasi-steady models accounting for these non-linear features have been proposed in the literature (Cummings 1984). More recently, an extensive experimental analysis relying on microphone measurements has been performed by Tonon et al (2013) in order to better account for such flow-induced effects in analytical models. Zhang and Bodony (2016) relied on direct numerical simulations (DNS) to further investigate the response of a simplified but realistic acoustic liner with subsonic grazing flow and high SPL tonal excitation.

While a significant body of work exists regarding the physics of isolated Helmholtz resonators, experiments aiming at characterizing the fluid mechanics of acoustic liners in complex situations (that is with significant turbulent grazing flow, large sound pressure levels and multi-harmonic excitation) are scarce: specific facilities are necessary and velocity measurement techniques with sub-millimeter resolution are required. Recently, Heuwinkel et al (2010) performed 2D phase-averaged Particle Image Velocimetry (PIV) above an acoustic liner to study the effects of both grazing and bias flows on an acoustic liner. A field of view of about $20 d \times 28 d$ 
with $d=2.5 \mathrm{~mm}$ was used, yielding a measurement resolution of about $720 \mu \mathrm{m}$. These results were compared with Laser Doppler Velocimetry (LDV) measurements performed on a $2 \mathrm{D}$ field of about $4 d \times 4 d$ above an orifice with a spatial resolution of about $100 \mu \mathrm{m}$, demonstrating that such optical measurement techniques are suited for acoustic liner studies in complex conditions. More recently, Haufe et al (2014) employed Doppler Global Velocimetry (DGV) to analyze the flow field above an acoustic liner with bias flow, while Gürtler et al (2016) improved this technique by using a high-speed camera. They showed that this measurement technique could be employed to measure velocity spectra in a plane above a liner orifice with a spatial resolution of about $300 \mu \mathrm{m}$. They furthermore quantified the energy transfer between the sound field and the flow.

These prior works have clearly demonstrated the applicability of optical measurement techniques to investigate the flow field details above acoustic liners. However, no systematic experimental analysis of the nearwall aerodynamic response of such resonators exploring the effects of the SPL forcing, the grazing flow velocity or the tonal content of the excitation have been reported to the knowledge of the authors. The purpose of the present work is thus to provide and analyze detailed velocity measurements above a conventional acoustic liner, obtained using a high-magnification PIV setup designed to reach fine spatial resolutions, for aerodynamic and acoustic conditions where linear and nonlinear behaviors are observed. This study intends to provide flow velocity measurements, rather than acoustic characteristics of a liner, that could serve to improve acoustic liner aerodynamic modeling in realistic conditions.

This article is organized as follows. First, details on the experiments performed are provided in Sect. 2, discussing the acoustic liner properties and the design of a high-magnification PIV setup. Second, results obtained for tonal excitation far from and near resonance are discussed in Sec. 3. Third, effects induced by variations in the SPL forcing and the aerodynamic conditions are presented in Sec. 4 for a multi-tonal excitation. The conclusions of this work are finally summarized in Sec. 5

\section{Experimental setup}

\subsection{Aero-thermo-acoustic duct}

The experiments were performed in the aero-thermoacoustic duct B2A at ONERA Toulouse. This facility, schematically shown in Fig. 2, is designed to study the acoustic and aerodynamic response of liners with grazing subsonic flows. This duct is a made of a $50 \mathrm{~mm} \times 50 \mathrm{~mm}$

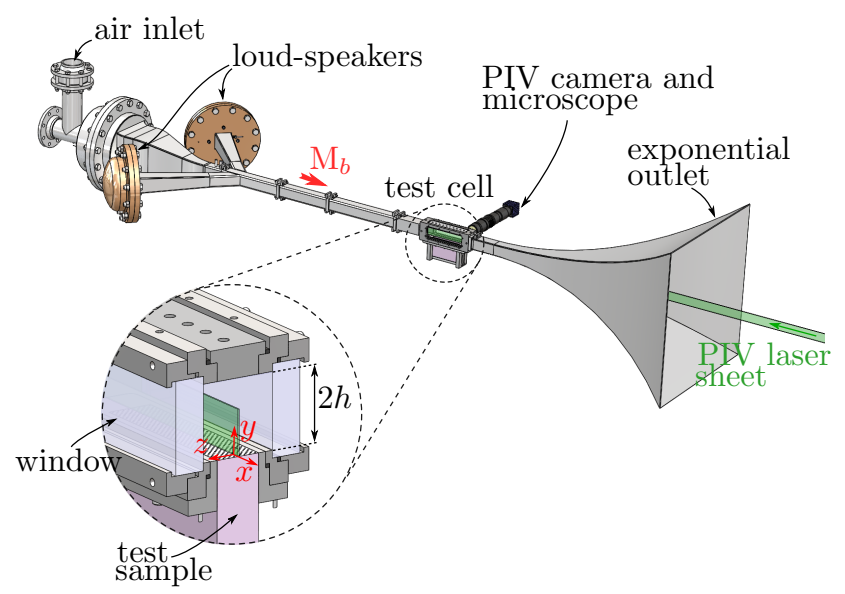

Fig. 2 Schematic of the aero-thermo-acoustic duct B2A at ONERA Toulouse illustrating the 2D high-magnification PIV setup used for the present study (long-distance microscope, PIV camera and constant-height laser sheet propagating from downstream); the inset shows a cross-section of the test-cell.

stainless steel square tube and acts as a waveguide with a cut-off acoustic frequency of $3450 \mathrm{~Hz}$ at ambient temperature. The $200 \mathrm{~mm}$-long test cell is equipped with two opposing silica windows for optical access. The acoustic liner is mounted in the lower wall of this test-section, centered spanwise, and has an area of $150 \mathrm{~mm} \times 30 \mathrm{~mm}$. The flow developing in the duct is in a fully turbulent state, with axial velocity fluctuations on the centerline having r.m.s. amplitudes of about $4.5 \%$ of the bulk velocity $U_{b}$. The duct termination is made quasianechoic using an exponential outlet connected to a muffled chamber, yielding acoustic reflection coefficients smaller than 0.2 for frequencies larger than $500 \mathrm{~Hz}$. Finally, plane acoustic waves propagating in the duct are generated by two acoustic drivers located $1.3 \mathrm{~m}$ upstream of the test section and placed symmetrically on each side of the duct. These acoustic drivers are used to generate pure tones and multi-sine signals with acoustic levels up to $150 \mathrm{~dB}$.

For the present experiment, the flow was regulated at a static temperature $T_{0}=20(1)^{\circ} \mathrm{C}$ and the massflow-rate $\dot{m}$ was varied between $50(1) \mathrm{g} / \mathrm{s}$ and $250(1) \mathrm{g} / \mathrm{s}$, corresponding to bulk Mach numbers $\mathrm{M}_{b} \equiv U_{b} / c_{0}$ ranging from 0.05 to 0.24 under normal atmospheric conditions. A speed of sound $c_{0}$ of about $343 \mathrm{~m} \mathrm{~s}^{-1}$ is considered. Using $2 h=50 \mathrm{~mm}$ as the characteristic length of the duct cross-section, a bulk Reynolds number $\mathrm{R}_{b} \equiv$ $2 U_{b} h / \nu$ is formed, with $\nu=1.51 \times 10^{-5} \mathrm{~m}^{2} \mathrm{~s}^{-1}$ the kinematic viscosity of air at $T_{0}$. This bulk Reynolds number was thus varied between $5.5 \times 10^{4}$ and $27.6 \times 10^{4}$ in the present experiments as listed in Tab. 1.

All the results are expressed in a right-handed cartesian reference frame such that the $x$-axis is oriented streamwise and the $y$-axis is vertical as represented in 
Fig. 1 and Fig. 2. The origin $O$ is located on the liner surface, approximately on the wall bisector, such that a liner orifice is tangent to the $z$-axis with its center located at $x=d / 2$. The velocity components along these axis $x, y$ and $z$ are respectively noted $u, v$ and $w$. Capital letters refer to (ensemble) mean velocity components and primes refer to r.m.s. velocity amplitudes.

\subsection{Description of the acoustic liner}

The acoustic liner used for this study is a conventional perforate-over-honeycomb single-layer liner, referred to as a Single Degree of Freedom (SDoF) liner in the literature. Its geometrical characteristics displayed in Fig. 1 are as follows: the orifices have a diameter $d=1.1 \mathrm{~mm}$; the perforated facesheet has a porosity $\sigma=\pi d^{2} /\left(2 L_{x} L_{z}\right)$ $=0.1$ and a thickness $\ell^{*}=0.8 \mathrm{~mm}$; the cavities are hexagonal honeycombs of height $H=30 \mathrm{~mm}$ with an inscribed circle diameter $D=9.6 \mathrm{~mm}$. The cross-sectional area of a honeycomb cell is then $S_{h}=\sqrt{3} D^{2} / 2$. The mean number of orifices per honeycomb cell is thus $N=\sigma S_{h} / S_{n}=8.4$ where $S_{n}=\pi d^{2} / 4$ is the orifice area.

For an isolated acoustic resonator with a single opening, the fundamental resonance frequency $\omega_{0}=2 \pi f_{0}$ can be estimated relying for example on the relation derived by Rienstra and Singh (2018)

$\kappa_{0} \tan \kappa_{0}=\frac{H S_{n}}{\ell S_{h}} \quad$ with $\kappa_{0}=\frac{\omega_{0} H}{c_{0}}$,

and where $\ell=\ell^{*}+\delta_{1}+\delta_{2}$ is the effective orifice neck length. This length is the sum of the geometrical neck length $\ell^{*}$ and two end correction terms $\delta_{i \in[1,2]} \approx 0.85$ $\left(S_{n} / \pi\right)^{1 / 2}\left(1-1.25 \xi_{i}\right)$ that account for the inertia of air on both sides of the facesheet, at the inner and outer apertures respectively (Ingard 1953), with $\xi_{1}=d / D$ and $\xi_{2}=0$. Accounting for the presence of $N$ orifices per honeycomb cell and changing the corresponding boundary condition in the derivations of Rienstra and Singh (2018), the relation Eq. (2) may be rewritten as $\kappa_{0} \tan \kappa_{0}=\frac{\sigma L}{\ell}$.

It is assumed here that the $N$ orifices per cell do not interact in a significant manner and do not alter the resonator dynamics. A discussion on the appropriate mass end corrections to apply to account for these interactions is provided by Ingard (1953): for the present liner geometry, it was estimated that such interactions between two holes could be neglected at first order. Relying on Eq. (3), one finally gets the resonance frequency $f_{0} \approx 1850 \mathrm{~Hz}$.

Experimentally, the absorption coefficient $\alpha$ of a liner without grazing flow and for normal incident acoustic waves (Eq. (1)) can be estimated in an impedance

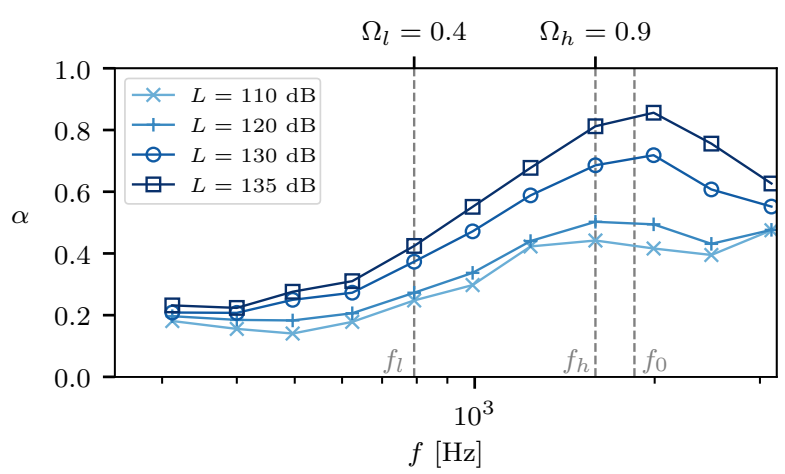

Fig. 3 Absorption coefficient $\alpha$ of the acoustic liner as a function of the frequency $f$ of the normal incident acoustic wave and for several sound pressure levels per tone $L$; measurements performed with no grazing flow and using a multisine signal composed of 11 pure tones, with frequencies that are far from $(\Omega<1)$ and near $(\Omega \approx 1)$ the liner resonance frequency $f_{0}$.

tube using two-point flush-mounted microphone measurements and a wave-sorting procedure (Bodén and Åbom 1986). This method was used with a multi-sine acoustic wave composed of 11 pure tones of equal intensity geometrically distributed over the frequency bandwidth response of the liner and for 4 increasing sound pressure levels (SPL) per tone. This geometric distribution is given by $\omega_{i+1}=\omega_{i} \times 10^{0.1}$ with $\omega_{1}=2 \pi \times$ $312 \mathrm{rad} \mathrm{s}^{-1}$. The resulting absorption coefficient distributions are given in Fig. 3. The two distinct response regimes of the resonator discussed in Sec. 1 can be identified in these results. The two lowest sound pressure levels $(110 \mathrm{~dB}$ and $120 \mathrm{~dB})$ yield similar absorption coefficient distributions, representative of a linear regime in the liner response dynamics. The highest absorption coefficient measured is obtained for the frequency $f_{h}=1592 \mathrm{~Hz}$, which is close to the resonance frequency $f_{0}$ previously estimated. For the two highest sound pressure levels however $(130 \mathrm{~dB}$ and $135 \mathrm{~dB})$, significantly higher absorption coefficients are observed and a slight shift in the resonance frequency may be guessed. Such a behavior is the result of non-linear effects induced by vortex shedding around the orifices on both sides of the liner facesheet, thus playing a major role in the resonator dynamics.

The objective of the present experiment is to investigate the flow dynamics around an orifice of this liner in both the linear and the non-linear response regimes. Tonal acoustic excitation around the frequency $f_{h}=1592 \mathrm{~Hz}$ will be considered in Sec. 3 and the results will be compared to the ones obtained at a frequency $f_{l}=792 \mathrm{~Hz}$ that is far from resonance. Furthermore, sound pressure levels and bulk Mach number effects will be investigated in Sec. 4 on the case of a multi-tonal 


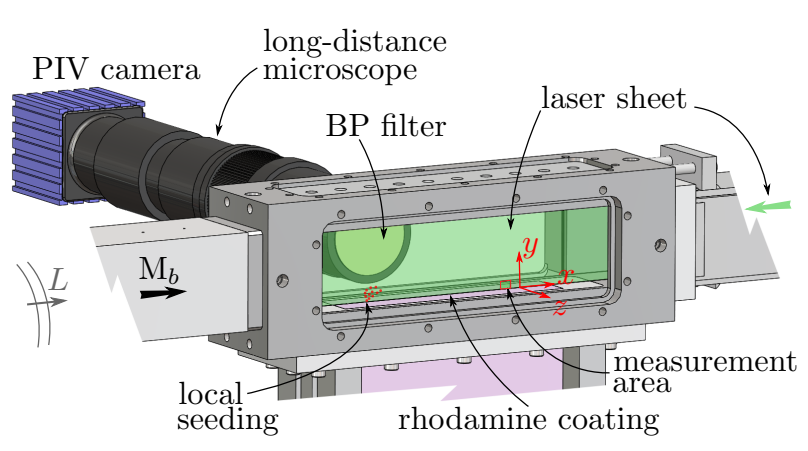

Fig. 4 Close-up of the PIV setup around the B2A $50 \mathrm{~mm} \times 50 \mathrm{~mm}$ duct; the PIV laser sheet coming from downstream is oriented in the $(x y)$ plane containing the vertical symmetry axis of the duct cross-section $(z=0)$; the flow is at a bulk Mach number $\mathrm{M}_{b}$ and the plane acoustic waves carried by the turbulent flow have individual levels $L$.

laser beam in the horizontal $(x z)$ plane laser beam in the vertical $(x y)$ plane

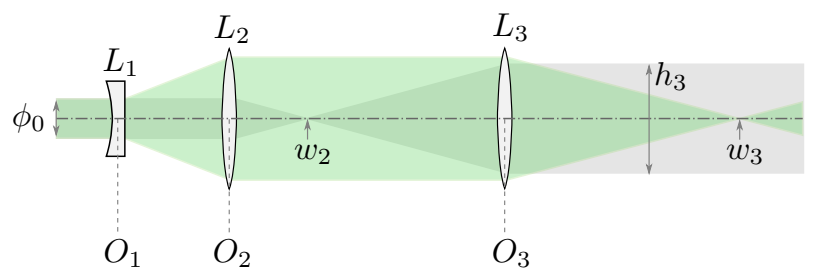

Fig. 5 Schematic of the optical setup used to generate the PIV laser-sheet; $\left(L_{1} L_{2}\right)$ forms a Galilean beam expander in the horizontal plane and $\left(L_{2} L_{3}\right)$ forms a Keplerian beam expander in the vertical plane; $L_{1}$ is a cylindrical lens of focal length $f_{1}=-100 \mathrm{~mm} ; L_{2}$ and $L_{3}$ are spherical lenses with $f_{2}=500 \mathrm{~mm}$ and $f_{3}=2000 \mathrm{~mm}$; the three lenses are separated by the distances $\overline{\mathrm{O}_{1} \mathrm{O}_{2}}=400 \mathrm{~mm}$ and $\overline{\mathrm{O}_{2} \mathrm{O}_{3}}=$ $2500 \mathrm{~mm}$; the laser beam diameter is $\phi_{0} \approx 6 \mathrm{~mm}$ with a divergence of about $1 \mathrm{mrad}$, yielding beam waists $w_{2}=0.5 \mathrm{~mm}$ and $w_{3}=0.4 \mathrm{~mm}$; the height of the final laser sheet is $h_{3}=24 \mathrm{~mm}$.

acoustic excitation similar to the one used to produce Fig. 3, thus featuring excitation near resonance.

\subsection{High-magnification PIV setup}

In order to perform non-intrusive measurements of the velocity fields close to an orifice of the liner with a high spatial resolution, a high-magnification planar Particle Image Velocimetry (PIV) setup was designed following the work of Kähler et al (2006). A schematic of the setup installed around the B2A duct test-cell is given in Fig. 2 and a close-up in Fig. 4. A double-pulse laser beam was generated using a Nd:YAG PIV laser (Quantel, BSL, $200 \mathrm{~mJ}$ per pulse) emitting at a wavelength $\lambda=532 \mathrm{~nm}$. This laser beam was shaped into a laser sheet using a set of three cylindrical and spherical lenses combined to form the optical arrangement schemati- cally displayed in Fig. 5. This optical setup provided a laser sheet of almost constant height $h_{3}=24 \mathrm{~mm}$, focused in the B2A test-cell over the acoustic liner with an approximate beam-waist $w_{3}=0.4 \mathrm{~mm}$, oriented in the ( $x y$ ) plane perpendicular to the liner surface and such that it contained a row of liner orifices along $z=0$. The objective of this optical setup was twofold: first, to generate a laser sheet in the $(x y)$ plane to perform 2D PIV measurements; second, to use almost parallel rays of light to avoid reflections that would prevent PIV measurements close to the liner surface. Using a laser sheet normally incident on the liner was not observed to meet this second requirement. This constant-height laser sheet also provides a high light intensity, that is necessary when working with small numerical apertures (typically around 0.1 here). In order to improve the signal-to-noise ratio and to reduce laser light reflections observed by the PIV camera, a thin layer of rhodamine paint was applied on the acoustic liner surface and a band-pass optical filter centered on $\lambda=532 \mathrm{~nm}$ and of $10 \mathrm{~nm}$ bandwidth was mounted on the camera lens. As shown in Fig. 6, almost no reflection is observed on the particle images while still providing illuminated particles very close to the surface.

These seeding particles were generated using a polydisperse aerosol generator (Topas ATM210) and DEHS oil (Di-Ethyl-Hexyl-Sebacat). According to the manufacturer, the mean diameter of the particles generated by this system is around $d_{p}=0.3 \mu \mathrm{m}$. A mean particle relaxation time can then be estimated by $\tau_{p}=$ $d_{p}^{2} \rho_{p} /\left(18 \mu_{0}\right) \approx 0.27 \mu \mathrm{s}$. The gas flow characteristic time scale may be estimated using the resonance frequency of the liner $1 / f_{0} \approx 540 \mu \mathrm{s}$, or by a more stringent time scale based on the maximum shear at a liner orifice $\tau_{g}=d / v_{\max }^{\prime} \approx 27 \mu \mathrm{s}$ where $d$ is the diameter of the liner orifices and $v_{\max }^{\prime} \sim 10 U_{\tau \max }$ is the maximum vertical velocity fluctuation measured in the present experiments (see Sect. 4). The Stokes number obtained $\mathrm{S}_{k}=\tau_{p} / \tau_{g} \approx 0.01$ is then sufficiently low to consider these particles as reliable flow tracers. As highlighted in Fig. 4, these particles were locally injected into the duct flow by filling one of the first upstream honeycomb cavities of the acoustic liner. This generally ensured a sufficiently dense seeding of the flow at the location where high-magnification PIV measurements were performed (approximately $100 \mathrm{~mm}$ downstream), although intermittent seeding could sometimes be observed in the top half part of the PIV images. As shown in Sect. 2.5, this protocol did not seem to introduce significant disturbances into the flow field.

The particles were imaged using a 4 MPx PIV camera (LaVision, SX4M) placed normally to the laser sheet and equipped with a long-distance microscope (Infinity 


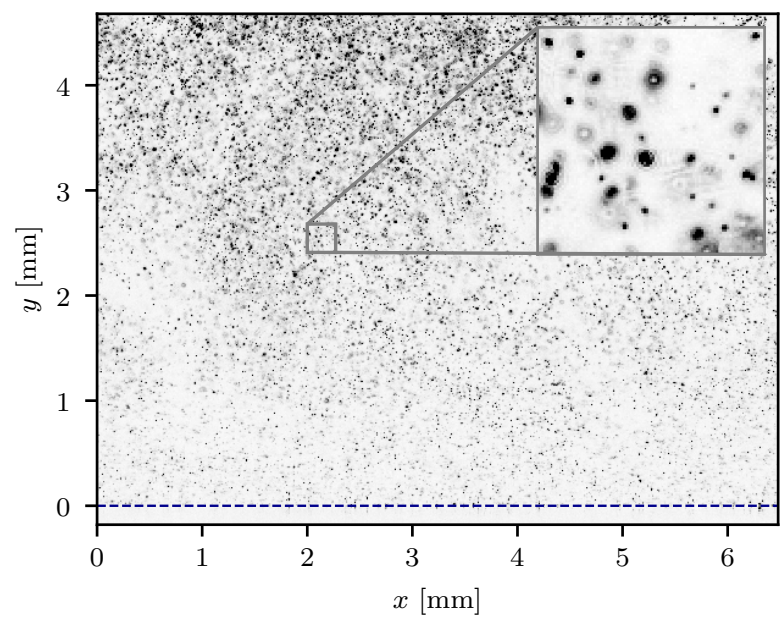

Fig. 6 Example of a particle image obtained with the highmagnification PIV setup and displayed using an inverted colormap; the dashed line highlights the location of the wall; the inset provides a close-up of images of particles.

K2 DistaMax with a CF-2 lens). A magnification factor of about 2 was obtained with a working distance of about $160 \mathrm{~mm}$, leading to dimensions of the measurement area of $4.9 \mathrm{~mm} \times 6.5 \mathrm{~mm}$ (that is $4.5 d \times 5.9 d$ ) and a spatial resolution of $2.74 \mu \mathrm{m} /$ pixel. The calibration of this setup was performed using a 2D precision calibration plate (Edmund Optics Grid Distortion Target 62-950) and a pinhole camera model, accounting for optical aberrations, typically leading to a r.m.s. calibration error of 0.3 pixel. The camera was focused on this calibration plate that was initially aligned on a row of liner orifices: the exact transverse location of the focusing plane is expected to lie within $\pm 0.1 \mathrm{~mm}$ with respect to the orifices centers. The depth of focus of this setup is approximately $d_{f}=40 \mu \mathrm{m}$, ten times smaller than the estimated laser sheet width $w_{3}$. As a consequence, illuminated particles that are not perfectly in the focusing plane are imaged with significant blur, yielding large bright particle images and dimmer ring-like patterns together with well focused particle images as shown in Fig. 6 . Such patterns and large particle images have been observed by Kähler et al (2012a) who also pointed-out the role of optical aberrations in these images. These out-of-focus particles are thus expected to play a significant role in the PIV correlation process, suggesting that the transverse spatial resolution of the measurement is likely larger than the exact depth of focus previously given: a conservative criterion could be to consider that the velocity fields presented in this study are the result of an intrinsic averaging over a width of $5 d_{f} \approx 0.2 \mathrm{~mm}$, that is approximately $0.18 d$ where $d$ is the liner orifices diameter.

\subsection{PIV acquisitions and post-processing}

A number of $N=1000$ PIV image pairs was acquired at a frequency of $10 \mathrm{~Hz}$ for every measurement sequence. The PIV double-pulse time delay was varied as a function of the bulk velocity such that a mean particle displacement of about $10 \mathrm{px}$ would be obtained. These time delays typically ranged from $0.3 \mu \mathrm{s}$ to $4 \mu \mathrm{s}$. Postprocessing of the particle images was performed using the ONERA in-house PIV software FOLKI-PIV (Champagnat et al 2011) allowing an accurate and fast evaluation of dense PIV velocity fields using Graphics Processing Units (GPUs) and an optical flow approach. An interrogation window size of $31 \mathrm{px}$ was used in the processing, leading to a spatial resolution of the measured velocity fields in the $(x y)$ plane of $85 \mu \mathrm{m}$, equivalent to $0.08 d$. A particular attention was given to the convergence of the algorithm and interrogation windows where the correlation score was lower than $30 \%$ were discarded in the computation of the flow statistics. It can be noted that a window-correlation approach was considered as sufficient enough for the present study aiming at analyzing the velocity fields near liner orifices, but that a finer spatial resolution could be attained relying on a Particle Tracking Velocimetry (PTV) approach as proposed by Kähler et al (2012b) for example.

As usual for PIV, the estimation of the measurement uncertainty is not trivial. Because of the high turbulence rate of the flows here studied, the velocity fluctuations were observed to be significantly larger than the random instantaneous uncertainties estimated relying on the work of Wieneke (2015). This implies that the global uncertainty of statistical quantities is dominated by the previous choice of the sample size $N$ (Sciacchitano and Wieneke 2016). To evaluate the statistical uncertainties associated with the mean and r.m.s. velocity fields measured, it is common to make a simplifying assumption of normally distributed velocities. In the present work, it was preferred to rely on a more general bootstrap method (Efron and Tibshirani 1994) in order to evaluate $95 \%$ confidence intervals (CI). Overall, for the most unfavorable cases showing large velocity fluctuations (a high bulk Mach number $\mathrm{M}_{b}=0.24$ or a large SPL excitation $L=140 \mathrm{~dB}$ ), the largest bounds of the $95 \%$ CI obtained on both mean and r.m.s. velocity results were of $\pm 0.8 \mathrm{~m} \mathrm{~s}^{-1}$. For the most favorable case (the no-liner case at a low bulk Mach number $\mathrm{M}_{b}=0.05$ ), bounds of about $\pm 0.2 \mathrm{~m} \mathrm{~s}^{-1}$ were evaluated. These estimated uncertainties are considered as satisfactory for the present discussion, the effects observed having significantly larger amplitudes. It can be noted however that in order to compare these 
PIV results with simulations one should consider in addition to these measurement uncertainties the filtering effect of the measurement process (induced by the planar and transverse spatial resolution) and the uncertainty on the exact location of the measurement plane with respect to the liner orifices among other sources of global uncertainty or error.

\subsection{Validation of the near-wall PIV measurements}

In order to evaluate the validity of the velocity measurements obtained with this PIV setup, preliminary tests over a rigid smooth wall were performed. Five bulk Reynolds numbers $\mathrm{R}_{b}$ listed in Tab. 1 were investigated. The mean axial velocity profiles obtained (averaged over the sample population and along the axial direction $x$ ) are given in Fig. 7 in wall-units, thus such that $U^{+} \equiv U / U_{\tau}$ and $y^{+} \equiv y U_{\tau} / \nu$, where $U_{\tau}$ is the friction velocity. On this figure are also plotted the law of the wall $U^{+}=y^{+}$and the logarithmic law, with $\kappa=0.42$ and $A=5.4$. These values for the log-law parameters are close to classical ones given for circular pipe flows (McKeon et al 2004) and were found to fit reasonably well the data for $y^{+}>70$. Nonetheless, a careful examination shows that slight deviations from this log-law appear for the largest values of $y / h$ which might be the result of a small velocity bias introduced by a scarcer and intermittent seeding in this region of the flow. Still, these deviations are minute and mainly appear away from the wall, where the influence of the acoustic liner will not be investigated. For validation of the data points in the buffer layer, a DNS result obtained by Pirozzoli et al (2018) for a turbulent flow in a square duct at a bulk Reynolds number $\mathrm{R}_{b}=4 \times 10^{4}$ is also represented in Fig 7 . As in the present experiment, this profile is taken along the duct wall bisector $(z=0)$ and is made dimensionless using a global mean friction velocity. Because of the lower bulk Reynolds number associated with this numerical result, the part of this profile representative of the wake region should not be expected to coincide with the data points obtained at higher values of $\mathrm{R}_{b}$.

A satisfactory collapse of all the measured mean axial velocity profiles is observed. Furthermore, these results compare favorably with the law of the wall for $y^{+}<8$, with the DNS profile in the buffer layer and with the log-law for $y^{+}>70$. For the lowest bulk Reynolds number, the first valid data point appears to be close to $y^{+}=3$. These results thus provide some confidence in the soundness of this PIV setup.

The estimates of the friction velocity $U_{\tau}$ required to provide the velocity profiles in inner variables shown in Fig. 7 were evaluated following two approaches. In

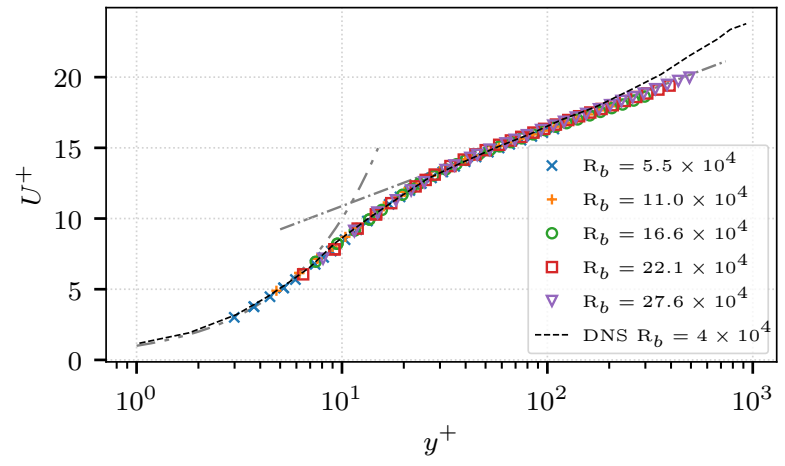

Fig. 7 Mean axial velocity profiles in wall units $U^{+}=f\left(y^{+}\right)$ measured by high-magnification PIV at five bulk Reynolds numbers $\mathrm{R}_{b}$ on a smooth wall in the B2A duct test-cell; - - - - : : $\log$-law $U^{+}=1 / \kappa \log \left(y^{+}\right)+A$ with $\kappa=0.42$ and $A=5.4$; $-\cdots$ - law of the wall $U^{+}=y^{+} ;---$: DNS result by Pirozzoli et al (2018) at $\mathrm{R}_{b}=4 \times 10^{4}$.

the first one, a Clauser-type method was considered, assuming a log-law in the mean axial velocity profiles with the Kármán and the additive constants $\kappa$ and $A$ previously given: a best fit to the data for $y^{+}>70$ provided an estimate for the parameter $U_{\tau}$. The values obtained following this approach are reported in Fig. 8 with open symbols. One issue with this method resides in the reduced extent of the log region in the present data and on the slight velocity bias previously observed for the largest $y$ values. In the second approach, we relied on Prandtl's friction law for smooth pipes that reads

$\frac{1}{\sqrt{f_{D}}}=2.0 \log _{10}\left(\mathrm{R}_{D_{h}} \sqrt{f_{D}}\right)-0.8$

where $f_{D}=4 C_{f}=8\left(U_{\tau} / U_{b}\right)^{2}$ is the average (Darcy) friction factor and $R_{D_{h}}$ is the Reynolds number based on the hydraulic diameter $D_{h}$. For square ducts, taking $D_{h}=2 h$ leads to satisfactory estimates of $U_{\tau}$ using Eq. 4 (Pirozzoli et al 2018). Refined estimates may be obtained using slight corrections that have been proposed in the literature (Jones 1976; Duan et al 2012), but they were not found to significantly modify the present results, nor was the use of a more recent version of the friction law for high Reynolds number flows (McKeon et al 2005). The estimates of the global friction velocities $U_{\tau}$ obtained with this second approach are reported in Fig. 8 using filled markers. As observed in this figure, the two methods provide values of $U_{\tau}$ that are in fair agreement, with relative differences lower than $\pm 5 \%$. In the following, all variables presented in wall-units will be evaluated using values of $U_{\tau}$ obtained with Prandtl's friction law. These estimated values are referred to as $U_{\tau 0}$ and are listed in Tab. 1. 


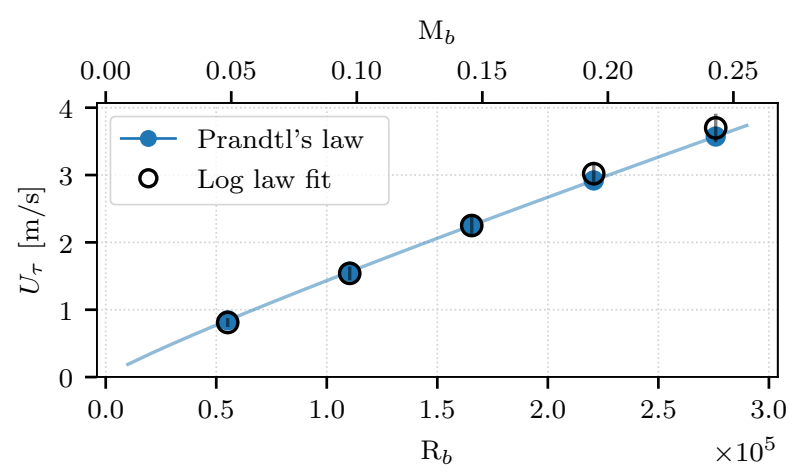

Fig. 8 Estimates of the mean friction velocity $U_{\tau}$ at five bulk Reynolds numbers $\mathrm{R}_{b}$ listed in Tab. 1 using two methods: (a) Prandtl's friction law and (b) a fit of the theoretical log-law defined in Fig. 7 on the mean axial velocity profiles (Clausertype method); for the last method, vertical error-bars with a relative amplitude of $\pm 5 \%$ are shown.

\section{Velocity fields near and far from resonance}

\subsection{No acoustic forcing}

Before analyzing the aerodynamic response of the liner to an acoustic excitation, details on the effect of the liner orifices on the near-wall flow with no acoustic forcing are presented. PIV measurements were performed above the acoustic liner mounted in the B2A duct testcell for the five bulk Reynolds numbers $\mathrm{R}_{b}$ listed in Tab. 1. These measurements, are compared with the smooth-wall case (Sect. 2.5) in Fig. 9, showing the mean axial velocity profiles in wall-units, with $U_{\tau}=U_{\tau 0}$, for two values of $R_{b}$. No appreciable differences between the smooth case and the liner case can be observed in this figure, suggesting that the present liner geometry does not induce any significant mean flow modification and that the friction velocity $U_{\tau}$ is not significantly altered. In terms of apparent surface roughness, this result shows that the Hama roughness function $\Delta U^{+}$ is close to zero for the flow conditions studied, within measurement uncertainty. Similarly, no significant differences were observed on the mean vertical velocity profiles $V(y)$ and on the fluctuations $u^{\prime}(y)$ and $v^{\prime}(y)$. This suggests that the self-generated oscillations of the separated shear-layers in the orifices composing this acoustic liner are not dynamically important, contrary to the isolated Helmholtz resonator cases experimentally studied by Ozalp et al (2003) and Ma et al (2009) using PIV.

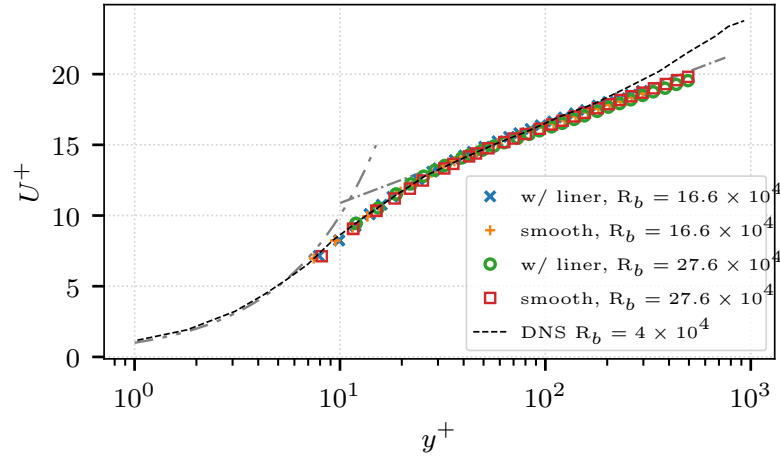

Fig. 9 Comparison of the mean axial velocity profiles in wall units $U^{+}=f\left(y^{+}\right)$(using the global friction velocity $\left.U_{\tau 0}\right)$ measured on a smooth wall and above the acoustic liner without acoustic forcing at two bulk Reynolds numbers $R_{b}$ (Tab. 1); refer to Fig. 7 for the definition of the dashed lines.

\subsection{Tonal excitation near and far from resonance}

The aerodynamic response of the liner is first analyzed for tonal excitation near and far from the resonance frequency $f_{0}$ estimated in Sect. 2.2. The objective of this section is to quantitatively illustrate the velocity fields above a liner orifice with a grazing flow in these two different response regimes. For this purpose, only one bulk Reynolds number $\mathrm{R}_{b}=5.5 \times 10^{4}$ (corresponding to $\mathrm{M}_{b}=0.048$ ) and one sound pressure level $L=132 \mathrm{~dB}$ are considered in this section. The effect of varying these two parameters will be described in Sect. 4. For the sound level $L$ selected, the liner is expected to provide an aerodynamic response characteristic of its nonlinear regime (in a no-flow configuration) as defined in Sec. 2.2.

As indicated in Fig. 3, the two forcing frequencies selected are $f_{l}=792 \mathrm{~Hz}$ and $f_{h}=1592 \mathrm{~Hz}$, respectively located far from and near the estimated resonance frequency of the liner. Introducing the frequency parameter $\Omega \equiv \omega / \omega_{0}$, one gets $\Omega_{l} \approx 0.4$ and $\Omega_{h} \approx 0.9$. Fig. 10 shows the mean and r.m.s. vertical velocity fields (resp. $V$ and $v^{\prime}$ ) obtained for the two forcing frequencies. These velocity amplitudes are made dimensionless using the global friction velocity $U_{\tau 0}$ defined in Sect. 2.5, even though the liner response is likely to change the value of the global friction velocity. This issue was not explored: the objective of this scaling is to provide a sense of the order of magnitude of the aerodynamic response of the liner with respect to the characteristic velocity scale at the wall. Indeed, following the work of Goldman and Panton (1976), it is believed that since in the present case the ratio $d / h=0.044 \ll 1$ the relevant velocity scale for the liner dynamics is more likely $U_{\tau}$ than $U_{b}$. 


\begin{tabular}{|lll|lllll|}
\hline $\begin{array}{l}\dot{m} \\
\left(\mathrm{~g} \mathrm{~s}^{-1}\right)\end{array}$ & $\begin{array}{l}U_{b} \\
\left(\mathrm{~m} \mathrm{~s}^{-1}\right)\end{array}$ & $\begin{array}{l}U_{\tau 0} \\
\left(\mathrm{~m} \mathrm{~s}^{-1}\right)\end{array}$ & $\begin{array}{l}C_{f 0} \\
\left(\times 10^{-3}\right)\end{array}$ & $\mathrm{M}_{b}$ & $\begin{array}{l}\mathrm{R}_{b} \\
\left(\times 10^{4}\right)\end{array}$ & $\begin{array}{l}\mathrm{R}_{\tau 0} \\
\left(\times 10^{3}\right)\end{array}$ & $d^{+}$ \\
\hline 50 & 16.6 & 0.84 & 5.12 & 0.048 & 5.5 & 1.39 & 61 \\
100 & 33.2 & 1.56 & 4.41 & 0.097 & 11.0 & 2.59 & 114 \\
150 & 49.8 & 2.25 & 4.08 & 0.145 & 16.5 & 3.74 & 164 \\
200 & 66.4 & 2.92 & 3.86 & 0.194 & 22.1 & 4.85 & 213 \\
250 & 83.0 & 3.57 & 3.70 & 0.242 & 27.6 & 5.93 & 261 \\
\hline
\end{tabular}

Table 1 Summary of the flow parameters as a function of the mass-flow-rate $\dot{m}=\rho U_{b} A$ set in the B2A square duct of cross-section $A=4 h^{2} ; U_{b}$ is the bulk velocity, $U_{\tau 0}$ the friction velocity evaluated using Prandtl's friction law (Eq. (4)), $C_{f 0}=2\left(U_{\tau 0} / U_{b}\right)^{2}$ is the friction coefficient based on $U_{\tau 0}, \mathrm{M}_{b}$ is the bulk Mach number, R $\mathrm{R}_{b} \equiv 2 h U_{b} / \nu$ is the bulk Reynolds number, $\mathrm{R}_{\tau 0} \equiv h U_{\tau 0} / \nu$ is the friction Reynolds number based on $U_{\tau 0}$ and $d^{+} \equiv d U_{\tau 0} / \nu$ is the liner orifice diameter in wall-unit.
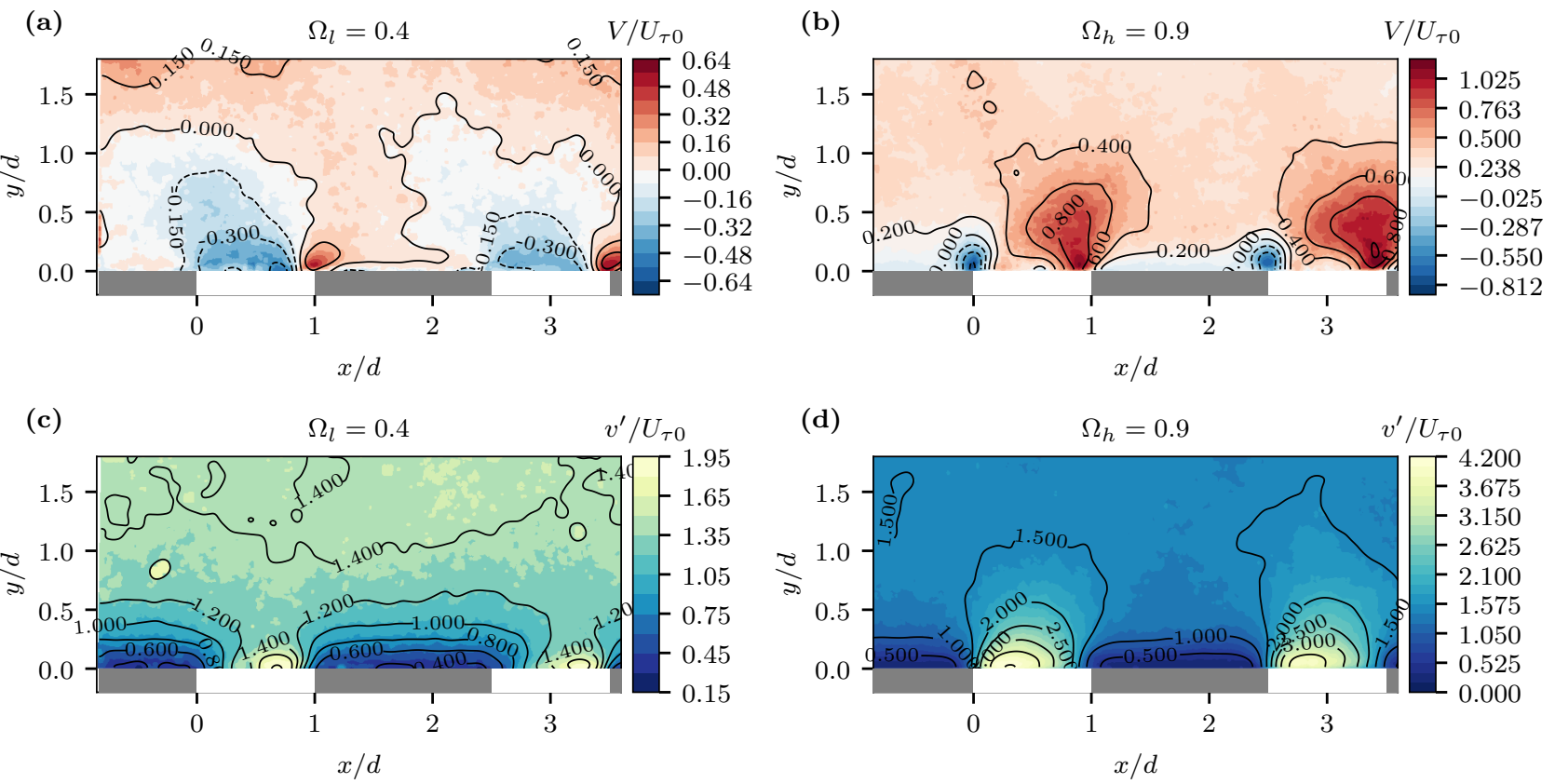

Fig. 10 Comparison of the vertical mean (first row) and r.m.s. (second row) velocity amplitudes above two orifices of the liner for two tonal acoustic excitation $f_{l}=792 \mathrm{~Hz}$ and $f_{h}=1592 \mathrm{~Hz}$ that are respectively far from $\left(\Omega_{l}=f_{l} / f_{0}=0.4\right)$ and near $\left(\Omega_{h}=f_{h} / f_{0}=0.9\right.$ ) the (no-flow) liner resonance frequency $f_{0}=1850 \mathrm{~Hz}$; results obtained with a grazing flow at $\mathrm{M}_{b}=0.048$ and made dimensionless using the global friction velocity $U_{\tau 0}$.

Away from resonance, for $\Omega=0.4$, the mean vertical velocity field (Fig. 10a) shows an extended region of negative velocities above the two orifices observed. A smaller region of positive vertical velocities follows downstream, imposed by the conservation of mass through an orifice. This average picture suggests a low to intermediate inflow/outflow regime as described by Baumeister and Rice (1975) and Tonon et al (2013). The vertical velocity fluctuation field shown in Fig. 10c suggests that the activity is mostly concentrated in the downstream half of the orifice section, with an amplitude two times larger than the no-liner friction velocity $U_{\tau 0}$.

Near resonance, for $\Omega=0.9$, the mean vertical velocity field depicted in Fig. 10b shows a more strongly polarized distribution compared to Fig. 10a, with a con- centrated region of negative velocity near the upstream edge of the orifices and a larger pocket of positive velocity near the downstream edge. In between, a core region of positive but almost null values is observed. This is representative of an intense inflow/outflow regime inducing large shear stresses at the orifice edges, with the formation of a synthetic jet that is advected downstream by the main flow. The vertical velocity fluctuations shown in Fig. 10d are more intense compared to the case $\Omega=0.4$ by a factor of two, with a peak amplitude located in the upstream half of the orifice section.

As pointed out by Zhang and Bodony (2016), the velocity scale of the in-orifice dynamics is not obvious in such a complex case where both the grazing flow and the acoustic forcing can play a role. A combined effect 
may be expected and several velocity scales may be considered (Goldman and Panton 1976): the bulk velocity $U_{b}$, the friction velocity $U_{\tau}$, the acoustic velocity of the incident sound field $p^{\prime} /\left(\rho_{0} c_{0}\right)$ and the sound-induced velocity of the resonator. Based on DNS, Zhang and Bodony (2016) proposed for their results the following velocity scale,

$\tilde{v}_{z} \equiv v_{a \infty}^{\prime}+u^{\prime}+0.6 U_{\tau}$,

where $u^{\prime}$ is the r.m.s. value of the axial velocity fluctuations (hypothetically transported into the orifice) and $v_{a \infty}^{\prime}$ is the sound induced in-orifice velocity amplitude defined by

$v_{a \infty}^{\prime} \equiv \frac{p^{\prime}}{\rho_{0} \omega \ell}$.

The incident acoustic pressure r.m.s. amplitude $p^{\prime}$ is related to the sound pressure level $L$ (in $\mathrm{dB}$ ) by

$p^{\prime}=p_{\text {ref }} 10^{L / 20}$

with $p_{\text {ref }}=20 \mu \mathrm{Pa}$. The definition provided by Eq. (6) is actually a high-frequency limit deduced from a lumpedelement model of a Helmholtz resonator with no grazing flow (see Morse et al (1948) for example) that reads

$v_{a}^{\prime}=\frac{p^{\prime}}{\rho_{0} \omega \ell} \frac{1}{\sqrt{\left[\left(\frac{\omega_{0}}{\omega}\right)^{2}-1\right]^{2}+\left(\frac{\omega_{0}}{\omega Q}\right)^{2}}}$.

In this expression $Q \equiv \omega_{0} / \gamma$ is the resonator quality factor that is inversely proportional to the damping coefficient $\gamma$ of the system and thus to the orifice resistance (Ingard 1953). The present value of the $Q$-factor is not precisely known and is most likely a function of the tangential flow velocity since it is well known that a grazing flow generally increases a liner resistance due to a reduction of the effective orifice area (Rogers and Hersh 1976). A typical value for such a resonator with no grazing flow is about 10 . Non-linear effects that are induced by a high SPL and a grazing flow will significantly increase the orifice resistance and thus decrease the value of $Q$ by typically a factor 10 , yielding $Q \approx 1$. We may then expect such a value to have the correct order of magnitude and to change with increasing values of $U_{\tau}$.

The velocity scales obtained using Eq. (5), Eq. (6) and Eq. (8) are summarized and compared in Tab. 2 to the velocity fluctuations measured and noted $v_{\text {max }}^{\prime}$ : a unit ratio indicates similar amplitudes. For the present study, relying on the velocity scale defined in Eq. (5) leads to over-estimated sound-induced in-orifice velocity amplitudes for the two frequencies considered. For the case far from resonance $(\Omega=0.4)$, as one could expect, the high-frequency limit given by Eq. (6) is inadequate, whereas for the case near resonance $(\Omega=0.9)$ the contributions associated with a grazing flow effect do not seem as relevant as in the work of Zhang and Bodony (2016) who mainly considered high-frequency excitation. Relying on Eq. (8), however, appears to provide satisfactory estimates in both cases for a value of $Q$ set to 1 , suggesting that this velocity scale estimate is appropriate for the present results.

We finally note that the recent work of Rienstra and Singh (2018) that intends to solve the non-linear system of equations for a Helmholtz resonator without grazing flow might also be used in order to estimate the velocity amplitudes at the orifice. This was not performed here for conciseness.

\subsection{Phase-averaged velocity fields}

Still considering the case of a bulk Mach number $\mathrm{M}_{b}=$ 0.048 with a tonal excitation at $L=132 \mathrm{~dB}$, phaselocked PIV measurements were performed at 8 phase angles $\phi$ with respect to the harmonic signal driving the loud-speakers in order to provide a mean description of the near-wall liner response for the two forced cases $\Omega=0.4$ and $\Omega=0.9$. Mean (phase-averaged) vertical velocity fields, referred to as $V_{\phi}$, at four phase angles are shown in Fig. 11, illustrating the inflow and outflow phases for the case near resonance. Note that the phase reference is arbitrary and that it was adjusted such that $\phi=0$ corresponds approximately to the beginning of the inflow cycle at the orifice considered. The outflow phase is characterized by a velocity amplitude larger than the inflow one, resulting in an asymmetry of the in-orifice flow dynamics, consistent with the slight positive values observed in Fig. 10b above the orifice center. Such a biased in-orifice flow has been also reported in the work of Zhang and Bodony (2016) with DNS results obtained for high-frequency excitation $\Omega>2$ and clearly finds its origin in the asymmetry of the flow conditions on both sides of the orifice.

Velocity profiles extracted from these phase-averaged vertical velocity fields along an horizontal line located at $y=0.07 d$, that is close to the liner surface, are shown in Fig. 12 for the two forcing cases. Far from resonance (Fig. 12a), both the inflow and the outflow phases show peak velocities in the downstream half of the orifice section, which is consistent with the classical picture of a vena contracta induced by the grazing flow separating at the upstream orifice edge and reducing the effective orifice section (Rogers and Hersh 1976). Following Tonon et al (2013), this regime could be termed as a low to intermediate inflow/outflow regime. Near resonance however (Fig. 12b), the velocity profiles display a milder streamwise asymmetry suggesting that the vena contracta effect induced by the grazing flow is 


\begin{tabular}{cccccc}
\hline$\Omega$ & $\begin{array}{c}v_{\max }^{\prime} / U_{\tau 0} \\
(\mathrm{PIV})\end{array}$ & $\begin{array}{c}v_{a \infty}^{\prime} / v_{\max }^{\prime} \\
(\mathrm{Eq} .(6))\end{array}$ & $\begin{array}{c}\tilde{v}_{z} / v_{\max }^{\prime} \\
(\text { Eq. }(5))\end{array}$ & $\begin{array}{c}v_{a}^{\prime} / v_{\max }^{\prime} \\
(\mathrm{Eq} .(8))\end{array}$ & $v_{a}^{\prime} / U_{\tau 0}$ \\
\hline 0.4 & 1.95 & 4.60 & 6.34 & 0.91 & 1.77 \\
0.9 & 4.2 & 1.06 & 1.87 & 0.87 & 3.65 \\
\hline
\end{tabular}

Table 2 Comparison of in-orifice velocity scales for the case of tonal acoustic forcing far from and near resonance at $L=132 \mathrm{~dB}$ with a grazing flow at $\mathrm{M}_{b}=0.048$.

(a)

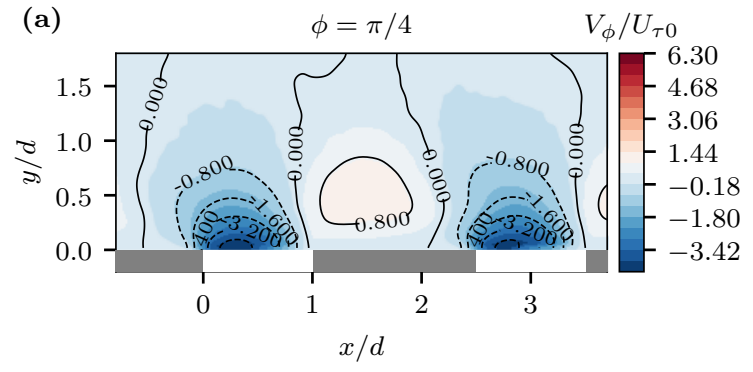

(b)

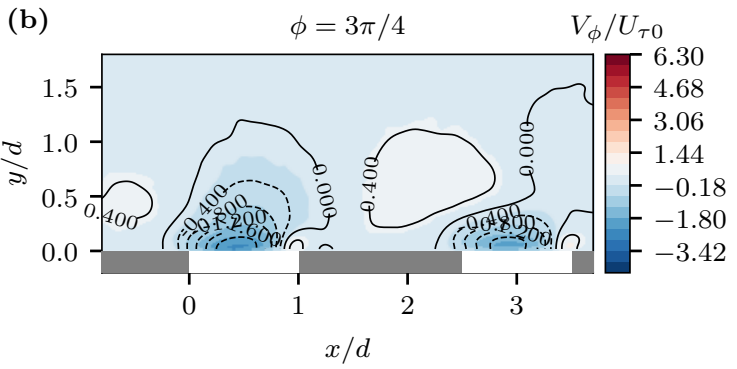

(c)

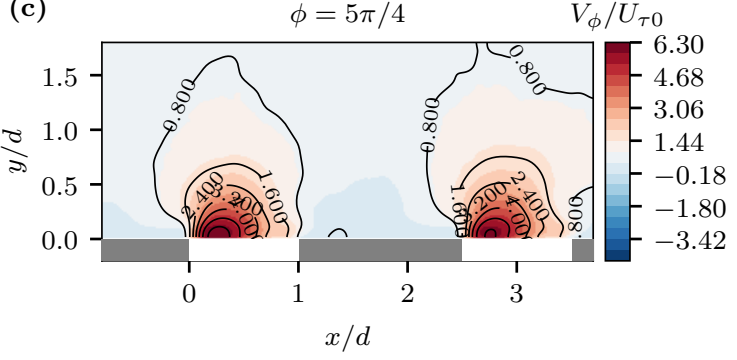

(d)

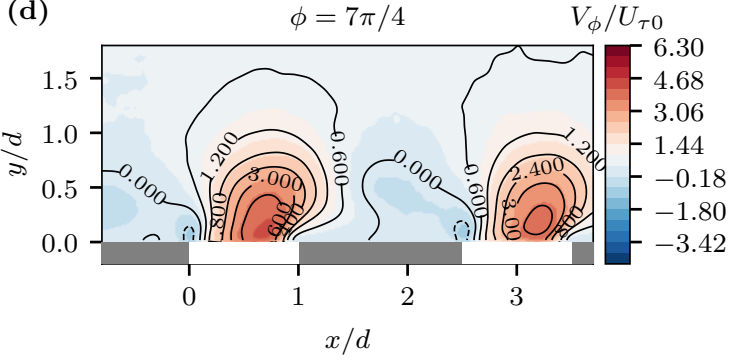

Fig. 11 Phase-averaged vertical velocity fields $V_{\phi}$ obtained for an acoustic forcing near resonance $(\Omega=0.9)$ with $L=$ $132 \mathrm{~dB}$ and a grazing flow at $M_{b}=0.048 ; \phi$ refers to the phase angle with respect to the acoustic excitation, such that $\phi=0$ corresponds approximately to the beginning of the inflow cycle.

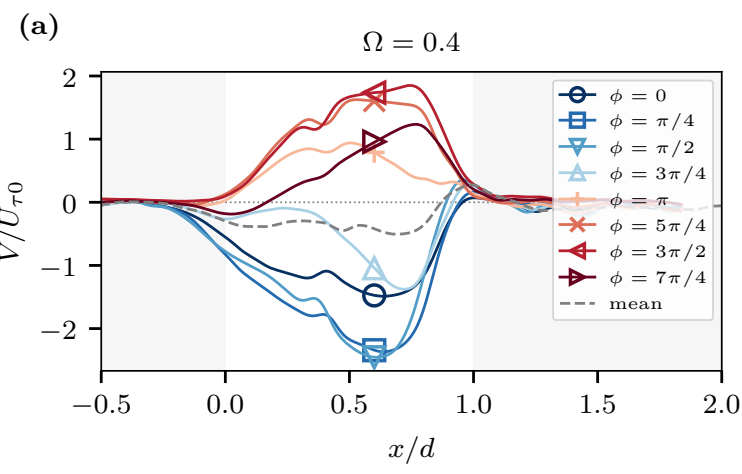

(b)

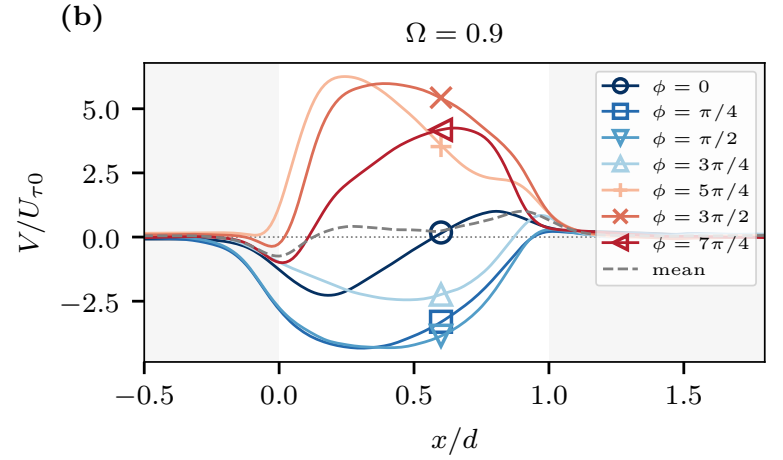

Fig. 12 Phase-averaged mean vertical velocity profiles $V / U_{\tau 0}$ extracted along an horizontal line located at $y / d=$ 0.07 above an orifice for the two forcing cases (a) far from and (b) near resonance, with $L=132 \mathrm{~dB}$ and $\mathrm{M}_{b}=0.048$.

less pronounced and that the in-orifice flow dynamics is mainly driven by the acoustic resonance. This high inflow/outflow regime approaches a pure bias flow situation, where vena contracta effects are rather associated with symmetric flow separation at the orifice edges.

These observations finally highlight that in order to satisfactorily model the in-orifice flow dynamics for such an acoustic liner in situations where typically $v_{\max }^{\prime} / U_{\tau 0}>$ 2 at the resonance, different inflow/outflow regimes need to be considered as a function of $\Omega$.

\section{Effect of $M_{b}$ and $L$ on the liner response to a multi-tonal excitation}

The liner response analyzed in the previous section highlighted some details of the flow dynamics for a specific set of global friction velocity $U_{\tau 0}$ (driven by $\mathrm{M}_{b}$ or $\mathrm{R}_{b}$ ) 
and sound pressure level $L$ at one forcing frequency. This section intends to provide details on the effect of varying these two parameters on the velocity field above an orifice. Furthermore, a multi-tonal excitation composed of the same 11 pure tones used in Sect. 2.2 is considered. Each tone is set to the same incident SPL that will be noted $L$ in the following: the overall SPL $L_{\mathrm{OA}}$ is then approximately equal to $L+10.4$. This choice is of interest for two main reasons. First, acoustic liners are usually employed in complex situations where multi-tonal excitation can be found, as in fan applications; it is not clear however whether a liner response depends on the spectral content of the excitation or not and quantitative data are required to explore this point. Second, to the knowledge of the authors, there are no results in the literature discussing the appropriate velocity scale to consider at a liner orifice under multi-tonal excitation. An attempt to address this last point is thus presented.

\subsection{Effect of the sound pressure level}

The mean and r.m.s. vertical velocity fields measured above an orifice for increasing values of SPL per tone $L \in[120,125,140] \mathrm{dB}$ are shown in Fig. 13, for a bulk Mach number $\mathrm{M}_{b}=0.048$. A fourth case corresponding to $L=132 \mathrm{~dB}$ is shown in the following Sect. 4.2 in Fig. 16 but is not reproduced here for conciseness. It can be observed that the lowest SPL per tone provides mean and r.m.s. velocity fields that are quite similar to the ones obtained for single-tone excitation near resonance in the previous section (see Fig. 10b and d). Increasing $L$ leads to progressively larger and more intense mean flow deformations. Vertical velocity fluctuations also significantly increase, up to a similar value of $v_{\max }^{\prime} / U_{\tau 0} \approx 8.5$ at the two highest $L$, as reported in Tab. 3. This observation is not fully explained, but two alternatives may be proposed: first, this might indicate an upper bound or a nonlinear limiting process; second, the two cases considered might yield resistance terms almost symmetrically located below and above the optimal one leading to maximum absorption, thus yielding similar in-orifice dynamics. This point requires further investigation with possible connections to make with the behavior of the acoustic liner impedance. A more quantitative picture is provided in Fig. 14 that shows r.m.s. velocity profiles extracted along a line close to the orifice at $y / d=0.07$. We note that these profiles may appear almost symmetric with respect to the orifice axis but a careful inspection shows that higher fluctuations are generally observed in the upstream half of the orifice section, suggesting here again an inflow/outflow activ-

\begin{tabular}{cccc}
\hline $\begin{array}{c}L \\
(\mathrm{~dB})\end{array}$ & $\begin{array}{c}v_{\max }^{\prime} / U_{\tau 0} \\
(\mathrm{PIV})\end{array}$ & $\begin{array}{c}\bar{v}_{a}^{\prime} / v_{\max }^{\prime} \\
(\text { Eq. }(9))\end{array}$ & $\bar{v}_{a}^{\prime} / U_{\tau 0}$ \\
\hline 120 & 3.3 & 0.64 & 2.12 \\
125 & 4.5 & 0.84 & 3.77 \\
132 & 8.7 & 0.97 & 8.44 \\
140 & 8.4 & 2.52 & 21.20 \\
\hline
\end{tabular}

Table 3 Measured vertical r.m.s. velocity amplitudes $v_{\max }^{\prime}$ above an orifice compared with the velocity amplitude estimate $\bar{v}_{a}^{\prime}$ given by Eq. (9) with an acoustic excitation composed of 11 pure tones, for an increasing SPL per tone $L$ and for $\mathrm{M}_{b}=0.048$.

ity more intense in this region for the present aerodynamic condition.

As observed in Fig. 14 for $L=132 \mathrm{~dB}$, the r.m.s. vertical velocity amplitude of the resonator with a multitonal excitation is clearly larger compared to the singletone excitation case presented in Sec. 3.2. A simple way of modeling such a multi-tonal forcing consists in assuming that each tone contributes independently of the others to the vertical velocity variance at the orifice. The global r.m.s. velocity amplitude $\bar{v}_{a}^{\prime}$ induced by the multi-tonal excitation is then given by

$\bar{v}_{a}^{\prime}=\sqrt{\sum_{i} v_{a}^{\prime 2}\left(\omega_{i}\right)}$,

where one may use Eq. (8) to evaluate the velocity variance $v_{a}^{\prime 2}\left(\omega_{i}\right)$ induced by a tonal excitation at frequency $\omega_{i}$. To evaluate the validity of this velocity estimate, a single value of $Q=1$ is considered, as in Sec. 3.2, regardless of the frequency and of the SPL. Results obtained following this approach are summarized in Tab. 3. It can be observed that satisfactory orders of magnitude are obtained for $L=125 \mathrm{~dB}$ and $132 \mathrm{~dB}$, leading to ratios $\bar{v}_{a}^{\prime} / v_{\max }^{\prime}$ close to unity. We furthermore note that the value of $\bar{v}_{a}^{\prime} / U_{\tau 0}$ for the case $L=125 \mathrm{~dB}$ is similar to the one obtained in Sec. 3.2 with a tonal excitation near resonance at $L=132 \mathrm{~dB}$ : this is consistent with the similarity observed previously between the associated velocity fields. However, the lowest SPL per tone $L=120 \mathrm{~dB}$ leads to a low velocity estimate, while the highest case $L=140 \mathrm{~dB}$ provides an estimate that is too large. This may indicate that the value for $Q$ considered is too low for the former case and too high for the latter. This trend bears some physical sense since non-linearities appearing with increasing SPL tend to increase the orifice resistance and thus decrease the $Q$ factor.

These results shown in Tab. 3 also highlight that the aerodynamic response of the liner under these conditions is significant compared to the tangential flow, with vertical velocity amplitudes $v_{\max }^{\prime}$ easily larger than the main flow velocity scale at the wall $U_{\tau 0}$. To further explore the implications of this aerodynamic response on 
(a)

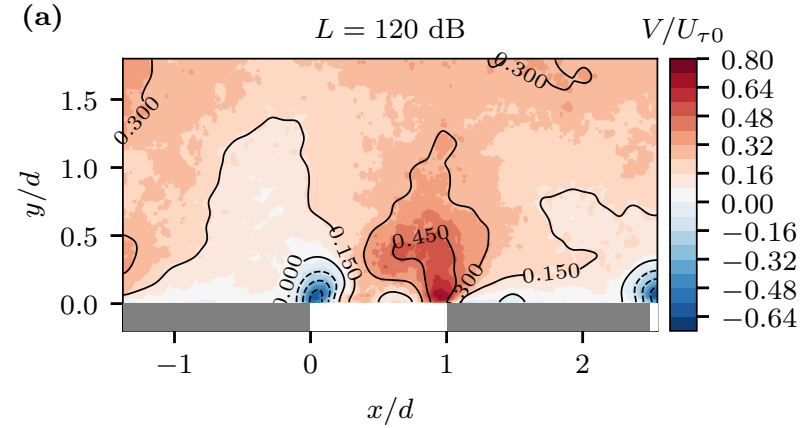

(c)

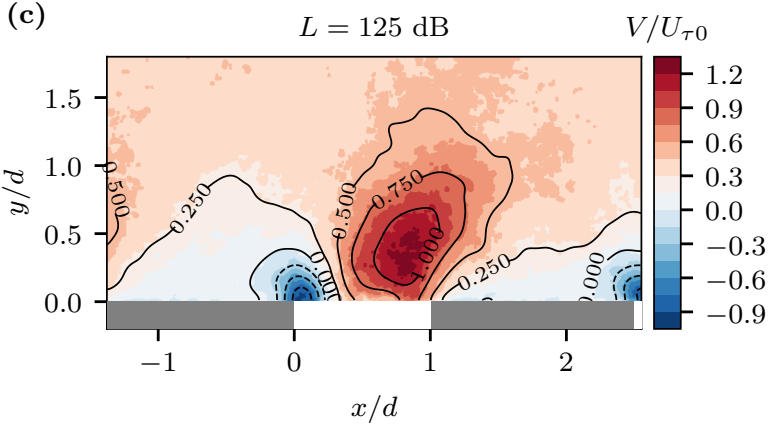

(e)

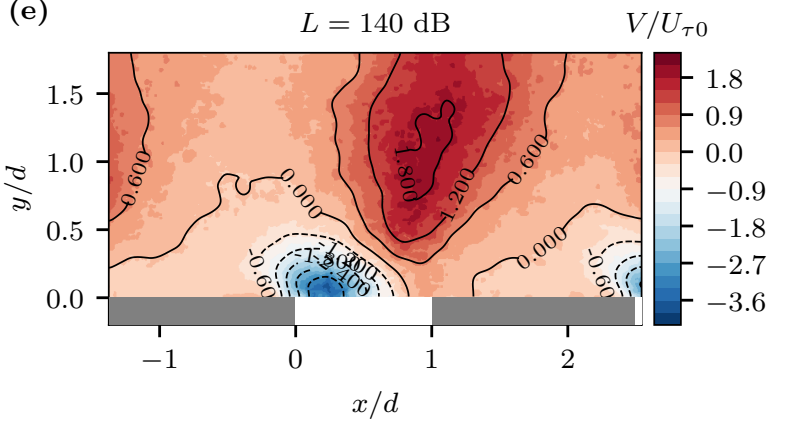

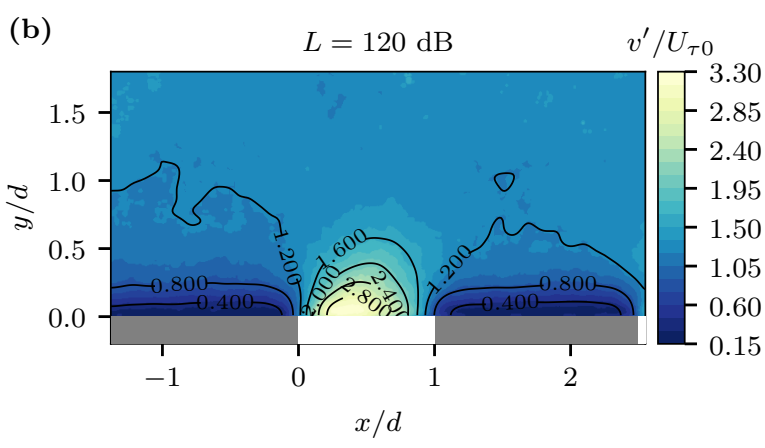
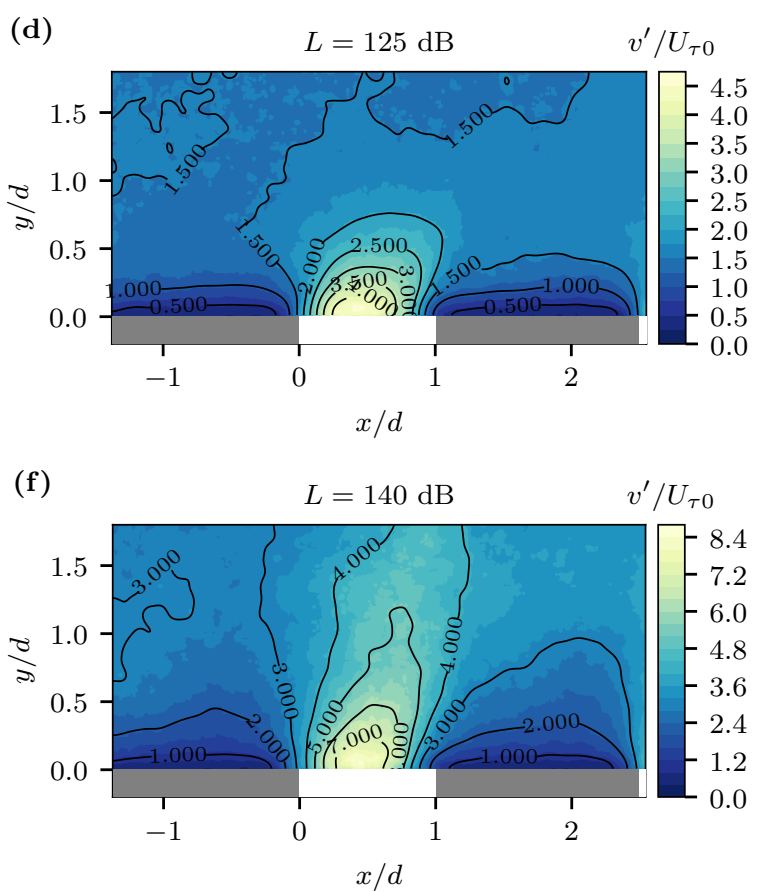

Fig. 13 Contours of mean (first column) and r.m.s. (second column) vertical velocity made dimensionless using the global friction velocity $U_{\tau 0}$ above an orifice of the liner at a bulk Mach number $\mathrm{M}_{b}=0.048$ and with multi-tonal excitation (11 pure tones with frequencies distributed as in Sec. 2.2) at three different sound pressure levels per tone $L$.

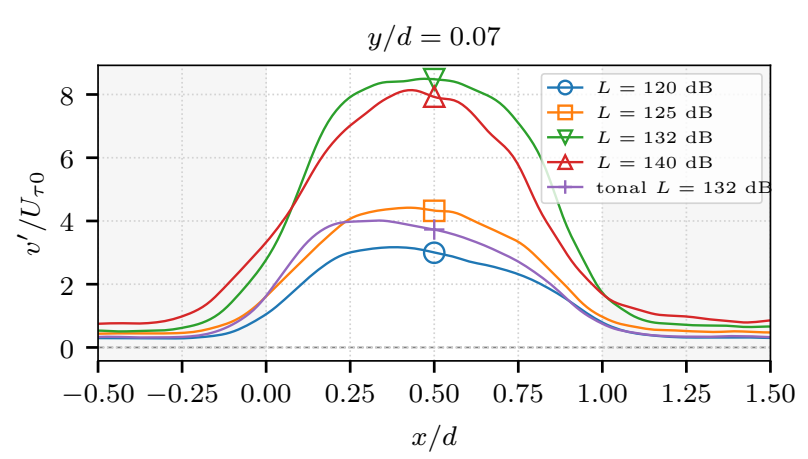

Fig. 14 Profiles of r.m.s. vertical velocity extracted from Fig. 13 along an horizontal line located at $y / d=0.07$; is also displayed the profile obtained for the single-tone excitation case shown in Fig. 10. the main flow, Fig. 15 shows the profiles of streamwiseaveraged axial and vertical mean velocity together with the no-liner case. This streamwise-averaging operation was performed over the length $L_{x}$ corresponding to the streamwise separation distance of two consecutive orifices. Examining Fig. 15a, one may attempt to draw a parallel with the aerodynamic effect of surface roughness for the two lowest values of $L(120 \mathrm{~dB}$ and $125 \mathrm{~dB})$ : the classical log-law appears to be vertically shifted by a quantity $\Delta U^{+}$that would be the Hama roughness function in roughness studies. We argue against this idea that a change of slope in the log region can be observed, more pronounced as $L$ is increased. Furthermore, significant distortions of both axial and vertical velocity profiles are seen for increasing values of $L$. This suggests that such a "rough-wall" analogy is not adequate, or at 
(a)

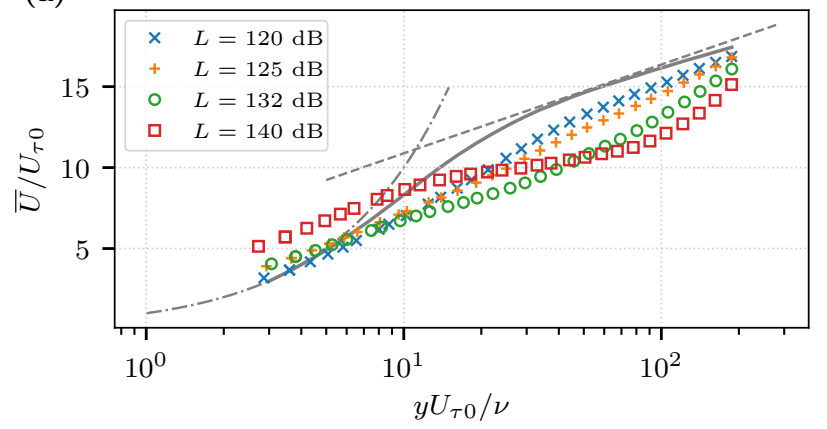

(b)

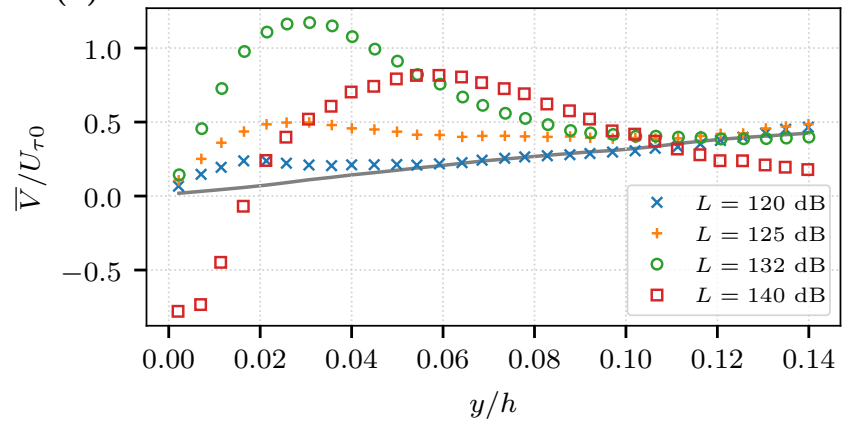

Fig. 15 (a) Streamwise-averaged mean axial $\bar{U}$ and (b) vertical $\bar{V}$ velocity profiles for 4 multi-tonal excitation levels $L$; these profiles are compared to the no-liner case (- $)$; in (a), are also displayed the law of the wall and the log-law as defined in Fig. 7 .

least significantly limited. It is thus concluded that, for the conditions here studied, the aerodynamic effect of a liner under acoustic excitation may not be accurately modeled relying on strategies like the equivalent sandgrain roughness (Jiménez 2004). Nonetheless, since the planar measurements analyzed here may provide an incomplete picture of the homogenization process, this issue may need to be further investigated by considering velocity profiles averaged over a periodic volume above a liner cell.

Finally, Fig. 15b displays the mean vertical velocity profiles $\bar{V} / U_{\tau 0}$. For the no-liner case, as a consequence of secondary motions in the duct corners, a linear increase starting from zero is observed along a wall bisector up to $y / h=0.14$, which is consistent with results reported in the literature (Pirozzoli et al 2018). Interestingly, an extended plateau of positive vertical velocity near the liner surface is obtained for the two cases $L=120 \mathrm{~dB}$ and $125 \mathrm{~dB}$. This observation suggests that a more suited analogy could then be made with a transpiration effect at the wall. This point is further developed in the next section.

\subsection{Effect of the bulk Mach number}

The effect of increasing the bulk Mach number $\mathrm{M}_{b}$ and thus the global friction velocity $U_{\tau 0}$ is analyzed in this section. The multi-tonal excitation case with $L=132 \mathrm{~dB}$ is considered and $\mathrm{M}_{b}$ is varied from 0.05 to 0.24 . Mean and r.m.s. vertical velocity maps are shown in Fig. 16, for only the first three Mach numbers for conciseness.

An increase of $\mathrm{M}_{b}$ yields a progressive reduction of the region of influence (or penetration length) of the sound-induced synthetic jet: the associated turbulent dynamics is progressively confined at the aperture and convected downstream, which is particularly evident on the r.m.s. velocity fields of Fig. 16. The vertical velocity fluctuations are swept and brought closer to the surface of the liner as $\mathrm{M}_{b}$ is increased. The location of maximum vertical velocity fluctuation at the aperture is also progressively moved from the upstream half of the orifice section to the downstream one. This suggests that separation at the upstream edge of the aperture has intensified, likely promoting a vena contracta effect in the orifice. This is particularly highlighted in Fig. 17a that shows r.m.s. vertical velocity profiles along the horizontal line $y / d=0.07$ : an almost symmetric profile is observed for $\mathrm{M}_{b}=0.05$ while for $\mathrm{M}_{b}=0.24$ it is significantly biased downstream.

As shown in Fig. 17a, the increase of the bulk Mach number does not change the order of magnitude of the vertical velocity fluctuations at the orifice. An increase of about $1 \mathrm{~m} \mathrm{~s}^{-1}$ is observed for $\mathrm{M}_{b}$ varying from 0.05 to 0.24 . We preferentially relate this trend to the vena contracta effect in the orifice that reduces the effective aperture cross-section, thus increasing the soundinduced velocity fluctuation amplitude in the orifice. Comparatively, it was observed (but not shown here) that the turbulence intensity of the main flow near the surface has increased by almost a factor 10: contrary to the work of Zhang and Bodony (2016), the present results indicate that if a turbulence-related velocity scale should be included in the in-orifice dynamics velocity scale previously defined by Eq. (9), a prefactor significantly lower than 1 should be considered.

While the amplitude of the in-orifice velocity fluctuations has not significantly increased with $\mathrm{M}_{b}$, the global velocity scale of the main flow at the wall $U_{\tau 0}$ has been multiplied by a factor of 4 . The ratio $v^{\prime} / U_{\tau 0}$ along the horizontal line at $y / d=0.07$ is represented in Fig. 17b, showing the reduced aerodynamic influence of the resonator for increasing $\mathrm{M}_{b}$ : the in-orifice velocity scale gradually becomes comparable with $U_{\tau 0}$. 
(a)

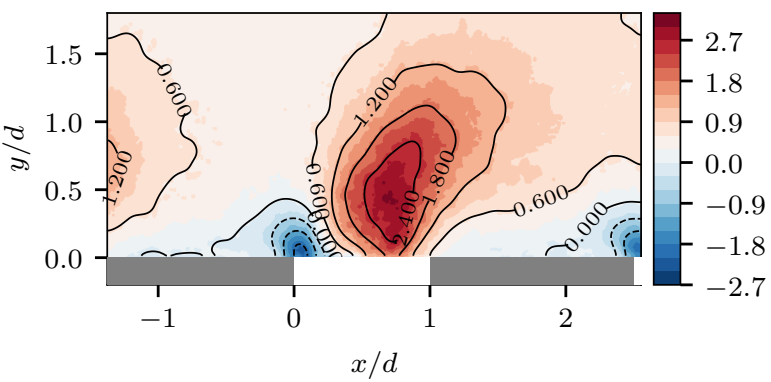

(c)

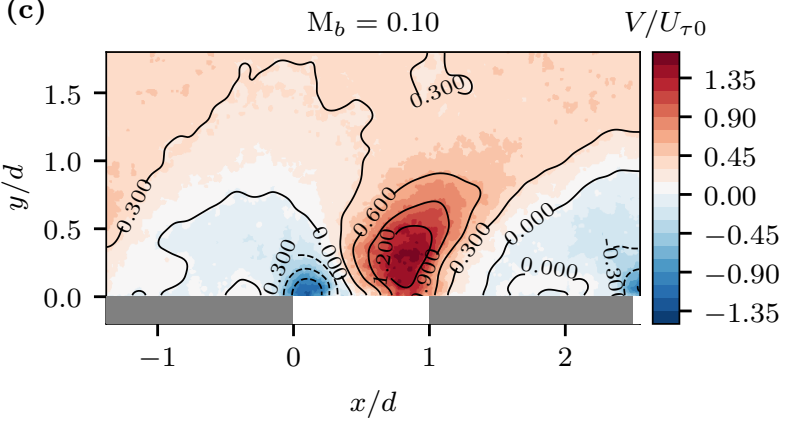

(e)

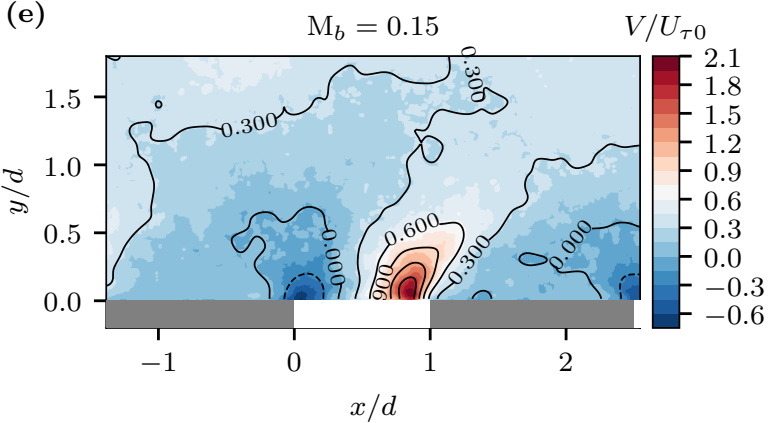

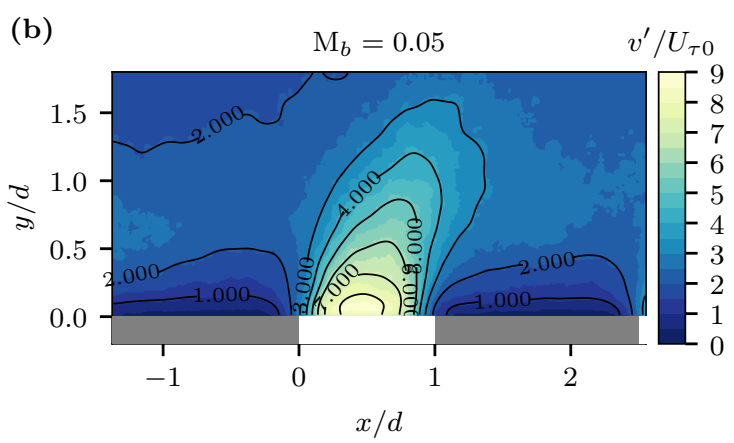

(d) $\quad \mathrm{M}_{b}=0.10 \quad v^{\prime} / U_{\tau 0}$
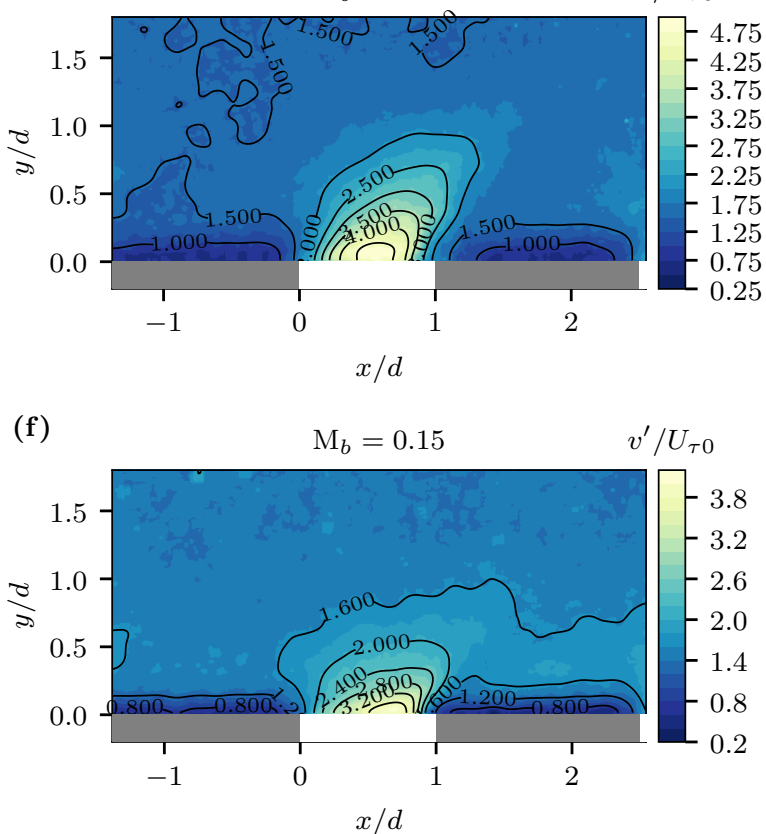

Fig. 16 Maps of mean (first column) and r.m.s. (second column) vertical velocity made dimensionless using the global friction velocity $U_{\tau 0}$ above an orifice of the liner for three bulk Mach numbers $\mathrm{M}_{b} \in[0.05,0.1,0.15]$; acoustic forcing is provided by a multi-tonal excitation (11 pure tones with frequencies distributed as in Sec. 2.2) with a sound pressure level per tone $L=132 \mathrm{~dB}$.
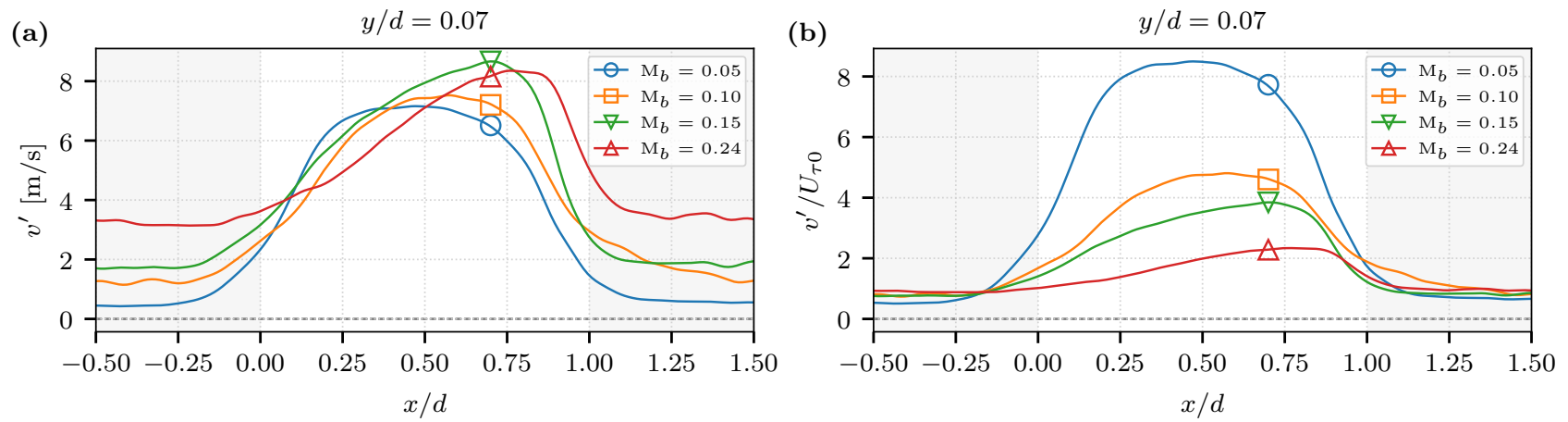

Fig. 17 Profiles of r.m.s. vertical velocity extracted from the PIV results shown in Fig. 16 along the horizontal line $y / d=0.07$ for 4 bulk Mach numbers $M_{b}$ (see Tab. 1) and for an acoustic excitation composed of 11 pure tones (see Sec. 2.2) individually set at a SPL $L=132 \mathrm{~dB}$; (b) is a dimensionless version of (a) using the global no-liner case friction velocity $U_{\tau 0}$. 
(a)

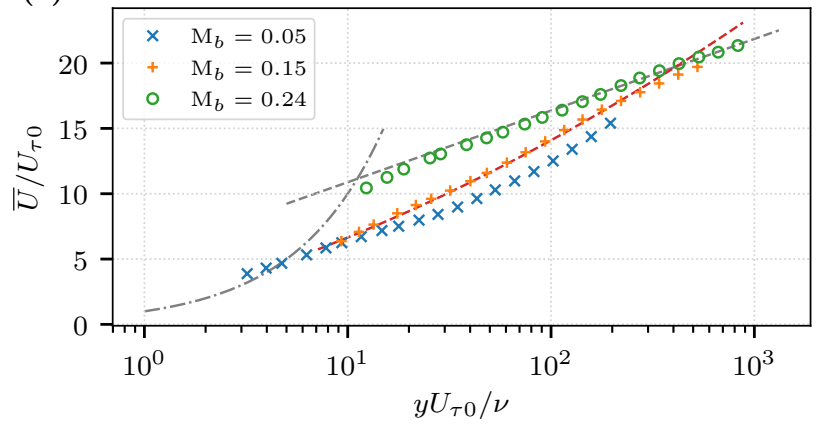

(b)

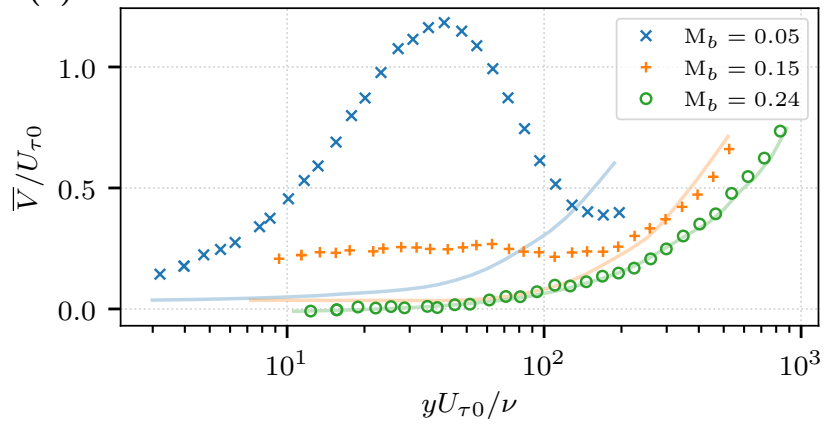

Fig. 18 Streamwise-averaged mean axial $\bar{U}$ (Fig. (a)) and vertical $\bar{V}$ (Fig. (b)) velocity profiles in wall units (using the noliner global friction velocity $U_{\tau 0}$ ) for three bulk Mach numbers $\mathrm{M}_{b}$ and for a multi-tonal acoustic excitation at $L=132 \mathrm{~dB}$; in Fig. (a) dashed gray lines represent the law of the wall and the log-law as defined in Fig. 7, and the dashed red line (- - ) is a fit to the $\mathrm{M}_{b}=0.15$ data using Eq. (10); in Fig. (b) the solid lines represent the no-liner case profiles (same colors used for the markers).

\begin{tabular}{cccc}
\hline $\mathrm{M}_{b}$ & $\begin{array}{c}v_{\max }^{\prime} / U_{\tau 0} \\
(\mathrm{PIV})\end{array}$ & $\begin{array}{c}\bar{v}_{a}^{\prime} / v_{\max }^{\prime} \\
(\text { Eq. }(9))\end{array}$ & $\bar{v}_{a}^{\prime} / U_{\tau 0}$ \\
\hline 0.05 & 8.7 & 0.97 & 8.44 \\
0.10 & 5.0 & 0.91 & 4.54 \\
0.15 & 4.1 & 0.77 & 3.15 \\
0.24 & 2.6 & 0.76 & 1.99 \\
\hline
\end{tabular}

Table 4 Measured vertical r.m.s. velocity amplitudes $v_{\max }^{\prime}$ above an orifice compared with the velocity amplitude estimate $\bar{v}_{a}^{\prime}$ given by Eq. (9) with an acoustic excitation composed of 11 pure tones at a SPL per tone $L=132 \mathrm{~dB}$ and for an increasing bulk Mach number $\mathrm{M}_{b}$.

This is highlighted in Tab. 4, where $v_{\max }^{\prime} / U_{\tau 0} \approx 2.6$ for $\mathrm{M}_{b}=0.24$. It is furthermore observed in Tab. 4 that the ratio $\bar{v}_{a}^{\prime} / v_{\max }^{\prime}$ obtained assuming $Q=1$ is still close to one, but moves away from unity with increasing $\mathrm{M}_{b}$. Analyzing this trend in terms of resistance behavior is more difficult than in Sec. 4.1: increasing the tangential flow velocity scale $U_{\tau 0}$ and thus intensifying the vena contract effects are known to increase the resonator resistance, but also to reduce the effective aperture area and the effective orifice neck length $\ell$ (Kirby and Cummings 1998), terms that play a significant role in Eq. (8). Such a discussion requiring further details on the acoustic properties of the resonator is however out of the scope of the present work and is not continued.

As in Sec. 4.1, the aerodynamic effect of the liner may be analyzed relying on streamwise-averaged mean axial and vertical velocity profiles, which are displayed in Fig. 18 in wall units and respectively referred to as $\bar{U}$ and $\bar{V}$. For the lowest bulk Mach number $\mathrm{M}_{b}=0.05$, clear differences exist compared to the no-liner case: the vertical velocity profile $\bar{V}$ shows a large peak near the wall yielding a significant deviation from the loglaw on the axial velocity profile $\bar{U}$. For the highest bulk Mach number $\mathrm{M}_{b}=0.24$, these mean velocity profiles are very similar to the no-liner case, suggesting that, in a spatially averaged manner, the liner response has no significant influence on the main flow. In-between, for $\mathrm{M}_{b}=0.15$, the average effect of the liner response translates to an almost constant vertical velocity component at the wall: as suggested in Sec. 4.1, an analogy in such an intermediate case may then be drawn with a uniform transpiration effect at the wall. As shown in the literature (Krogstad and Kourakine 2000), such a uniform injection leads to a modification of the classical log law found in turbulent boundary layers in such a way that

$U^{+}=U_{0}^{+}+\frac{1}{\kappa^{\prime}} \log \left(\frac{y^{+}}{y_{0}^{+}}\right)+\frac{V_{w}^{+}}{4}\left[\frac{1}{\kappa} \log \left(\frac{y^{+}}{y_{0}^{+}}\right)\right]^{2}$

where $\kappa^{\prime} \equiv \kappa / \sqrt{1+U_{0}^{+} V_{w}^{+}}, V_{w}$ is the mean injection velocity and $U_{0}^{+}$and $y_{0}^{+}$are integration constants. As shown in Fig. 18a, a satisfactory fit to the averaged axial velocity profile for $\mathrm{M}_{b}=0.15$ is obtained using this expression. The two lowest SPL cases shown in Fig. 15 and the tonal excitation case near resonance discussed in Sec. 3.2 were also found be correctly fitted by such an expression. It is then suggested that, under certain conditions, the aerodynamic effect of an acoustic liner may be satisfactorily modeled relying on such a "walltranspiration" analogy. An expected difficulty should then be found in the evaluation of the associated parameters that are the mean injection velocity and the equivalent friction velocity, which represents a complete study on its own.

Overall, based on the values of $v_{\max }^{\prime} / U_{\tau 0}$ obtained in this work and reported in Tab. 2, Tab. 3 and Tab. 4, it was observed that for about $v_{\max }^{\prime} / U_{\tau 0}<2$ no significant aerodynamic effect (in a streamwise average sense) was associated with the liner response; for $v_{\max }^{\prime} / U_{\tau 0}>$ 
5 large streamwise-average mean flow distortions appeared; in-between, for $2<v_{\max }^{\prime} / U_{\tau 0}<5$, the streamwiseaverage mean vertical velocity distribution was observed to be relatively uniform near the wall, suggesting that a wall-transpiration analogy might be relevant, while being obviously inexact. The bounds here proposed may be case-dependent and, as noted in Sect. 4.1, these conclusions are drawn from planar PIV measurements: future work should then consider numerical or experimental results obtained in a volume above a liner orifice.

\section{Conclusion}

This experimental work focused on the aerodynamic response of a conventional acoustic liner with a grazing flow. It was shown that a high-magnification PIV setup could be successfully used to measure the velocity dynamics near a millimeter-sized liner orifice. A systematic analysis of the effects of the forcing frequency, the forcing sound pressure level and the tangential flow velocity was then conducted.

The present results indicate different aerodynamic response regimes depending mainly on two parameters: the frequency parameter $\Omega=f / f_{0}$ and the ratio $v^{\prime} / U_{\tau}$. Acoustic excitation near resonance $(\Omega \approx 1)$ naturally yields to an intense aerodynamic response of the acoustic resonator, which may lead to in-orifice velocity scales $v^{\prime}$ much larger than the tangential velocity scale $U_{\tau}$. This behavior can thus profoundly alter the in-orifice aerodynamics with a large reduction of the vena-contracta effect induced by the grazing flow. Away from resonance, this vena contract effect is likely to play a more significant role.

The ratio of the near-orifice resonance-related velocity scale $v^{\prime}$ to the friction velocity $U_{\tau}$ was observed to provide a convenient way of categorizing the different cases in terms of their global aerodynamic effects. For low values, typically $v^{\prime} / U_{\tau}<2$, little changes were observed on the mean, streamwise-averaged velocity profiles. For large values, typically greater than 5, large mean flow distortions were observed. These are the result of synthetic jets formed at the liner orifices and able to penetrate deeply in the main flow. An intermediate regime was reported, for which a "transpiration wall" analogy rather than a "rough-wall" analogy appeared adequate. This point however requires further investigation and both numerical and experimental complementary analysis should be considered.

Finally, it was verified that a near-orifice velocity scale estimate based on a lumped-element method could be adequate for both tonal and multi-tonal acoustic excitation cases. One may then rely on such an estimate to evaluate $v^{\prime}$ for a specific case (given a SPL and an aerodynamic condition) and deduce a value for the ratio $v^{\prime} / U_{\tau}$ assuming a known friction velocity, then providing an estimate of the aerodynamic regime based on the above discussion. This approach lacks today some ingredients, such as an explicit and robust estimation of the $Q$-factor of the acoustic resonator that depends on the SPL and the vena contracta effects, and a confirmation of the relevance of the bounds proposed on $v^{\prime} / U_{\tau}$. Future work should then intend to clarify these points.

Acknowledgements This project has partly received funding from the Clean Sky 2 Joint Undertaking under the European Union's Horizon 2020 research and innovation program under grant agreement No. 681856 - ASPIRE. The authors wish to thank Delphine Sebbane for her technical assistance and expertise on the B2A duct and Airbus for manufacturing the acoustic liner.

\section{References}

Baumeister KJ, Rice EJ (1975) Visual study of the effect of grazing flow on the oscillatory flow in a resonator orifice. Tech. rep., NASA

Bodén H, Åbom M (1986) Influence of errors on the twomicrophone method for measuring acoustic properties in ducts. The Journal of the Acoustical Society of America 79(2):541-549, doi:10.1121/1.393542

Champagnat F, Plyer A, Besnerais GL, Leclaire B, Davoust S, Sant YL (2011) Fast and accurate PIV computation using highly parallel iterative correlation maximization. Experiments in Fluids 50(4):1169-1182, doi:10.1007/s00348011-1054-x

Charwat AF, Walker BE (1983) The velocity perturbations above the orifice of an acoustically excited cavity in grazing flow. Journal of Fluid Mechanics 128(-1):413, doi:10.1017/s0022112083000531

Cummings A (1984) Acoustic nonlinearities and power losses at orifices. AIAA Journal 22(6):786-792, doi: $10.2514 / 3.8680$

Duan Z, Yovanovich MM, Muzychka YS (2012) Pressure drop for fully developed turbulent flow in circular and noncircular ducts. Journal of Fluids Engineering 134(6):061,201, doi: $10.1115 / 1.4006861$

Efron B, Tibshirani RJ (1994) An introduction to the bootstrap. CRC press

Goldman AL, Panton RL (1976) Measurement of the acoustic impedance of an orifice under a turbulent boundary layer. The Journal of the Acoustical Society of America 60(6):1397-1405, doi:10.1121/1.381233

Guess A (1975) Calculation of perforated plate liner parameters from specified acoustic resistance and reactance. Journal of Sound and Vibration 40(1):119-137, doi:10.1016/s0022-460x(75)80234-3

Gürtler J, Haufe D, Schulz A, Bake F, Enghardt L, Czarske J, Fischer A (2016) High-speed camera-based measurement system for aeroacoustic investigations. Journal of Sensors and Sensor Systems 5(1):125-136, doi:10.5194/jsss-5-1252016

Haufe D, Pietzonka S, Schulz A, Bake F, Enghardt L, Czarske JW, Fischer A (2014) Aeroacoustic near-field measurements with microscale resolution. Measurement Sci- 
ence and Technology 25(10):105,301, doi:10.1088/09570233/25/10/105301

Heuwinkel C, Piot E, Micheli F, Fischer A, Enghardt L, Bake F, Röhle I (2010) Characterization of a perforated liner by acoustic and optical measurements. In: 16th AIAA/CEAS Aeroacoustics Conference, American Institute of Aeronautics and Astronautics, doi:10.2514/6.2010-3765

Ingard U (1953) On the theory and design of acoustic resonators. The Journal of the Acoustical Society of America 25(6):1037-1061, doi:10.1121/1.1907235

Ingard U, Ising H (1967) Acoustic nonlinearity of an orifice. The Journal of the Acoustical Society of America 42(1):617, doi:10.1121/1.1910576

Ingård U, Labate S (1950) Acoustic circulation effects and the nonlinear impedance of orifices. The Journal of the Acoustical Society of America 22(2):211-218, doi:10.1121/1.1906591

Jiménez J (2004) Turbulent flows over rough walls. Annual Review of Fluid Mechanics 36(1):173-196, doi:10.1146/annurev.fluid.36.050802.122103

Jones OC (1976) An improvement in the calculation of turbulent friction in rectangular ducts. Journal of Fluids Engineering 98(2):173, doi:10.1115/1.3448250

Kähler CJ, Scholz U, Ortmanns J (2006) Wall-shear-stress and near-wall turbulence measurements up to single pixel resolution by means of long-distance micro-PIV. Experiments in Fluids 41(2):327-341, doi:10.1007/s00348-0060167-0

Kähler CJ, Scharnowski S, Cierpka C (2012a) On the resolution limit of digital particle image velocimetry. Experiments in Fluids 52(6):1629-1639, doi:10.1007/s00348012-1280-x

Kähler CJ, Scharnowski S, Cierpka C (2012b) On the uncertainty of digital PIV and PTV near walls. Experiments in Fluids 52(6):1641-1656, doi:10.1007/s00348-012-1307-3

Kirby R, Cummings A (1998) The impedance of perforated plates subjected to grazing gas flow and backed by porous media. Journal of Sound and Vibration 217(4):619-636, doi:10.1006/jsvi.1998.1811

Krogstad PA, Kourakine A (2000) Some effects of localized injection on the turbulence structure in a boundary layer. Physics of Fluids 12(11):2990, doi:10.1063/1.1314338

Ma R, Slaboch PE, Morris SC (2009) Fluid mechanics of the flow-excited helmholtz resonator. Journal of Fluid Mechanics 623:1, doi:10.1017/s0022112008003911

McKeon BJ, Li J, Jiang W, Morrison JF, Smits AJ (2004) Further observations on the mean velocity distribution in fully developed pipe flow. Journal of Fluid Mechanics 501:135-147, doi:10.1017/s0022112003007304

McKeon BJ, Zagarola MV, Smits AJ (2005) A new friction factor relationship for fully developed pipe flow. Journal of Fluid Mechanics 538(-1):429, doi:10.1017/s0022112005005501

Melling T (1973) The acoustic impendance of perforates at medium and high sound pressure levels. Journal of Sound and Vibration 29(1):1-65, doi:10.1016/s0022$460 \mathrm{x}(73) 80125-7$

Morse PM, of America AS, of Physics AI (1948) Vibration and sound, vol 2. McGraw-Hill New York

Motsinger R, Kraft R (1991) Design and performance of duct acoustic treatment. In: Aeroacoustics of Flight Vehicles: Theory and Practice. Volume 2: Noise Control, vol 2

Ozalp C, Pinarbasi A, Rockwell D (2003) Self-excited oscillations of turbulent inflow along a perforated plate. Journal of Fluids and Structures 17(7):955-970, doi:10.1016/s0889-9746(03)00045-8
Pirozzoli S, Modesti D, Orlandi P, Grasso F (2018) Turbulence and secondary motions in square duct flow. Journal of Fluid Mechanics 840:631-655, doi:10.1017/jfm.2018.66

Rienstra SW, Hirschberg A (2018) An introduction to acoustics. Eindhoven University of Technology URL http: //www.win.tue.nl/ sjoerdr/papers/boek.pdf

Rienstra SW, Singh DK (2018) Nonlinear asymptotic impedance model for a helmholtz resonator of finite depth. AIAA Journal 56(5):1792-1802, doi: $10.2514 / 1 . j 055882$

Roche JM, Leylekian L, Delattre G, Vuillot F (2009) Aircraft fan noise absorption: DNS of the acoustic dissipation of resonant liners. In: 15th AIAA/CEAS Aeroacoustics Conference (30th AIAA Aeroacoustics Conference), American Institute of Aeronautics and Astronautics, doi:10.2514/6.2009-3146

Rogers T, Hersh A (1976) The effect of grazing flow on the steady state resistance of square-edged orifices. Aeroacoustics: Fan Noise And Control; Duct Acoustics; Rotor Noise 43

Sciacchitano A, Wieneke B (2016) PIV uncertainty propagation. Measurement Science and Technology 27(8):084,006, doi:10.1088/0957-0233/27/8/084006

Sivian LJ (1935) Acoustic impedance of small orifices. The Journal of the Acoustical Society of America 7(2):94-101, doi:10.1121/1.1915795

Tonon D, Moers E, Hirschberg A (2013) Quasi-steady acoustic response of wall perforations subject to a grazingbias flow combination. Journal of Sound and Vibration 332(7):1654-1673, doi:10.1016/j.jsv.2012.11.024

Wieneke B (2015) PIV uncertainty quantification from correlation statistics. Measurement Science and Technology 26(7):074,002, doi:10.1088/0957-0233/26/7/074002

Zhang Q, Bodony DJ (2012) Numerical investigation and modelling of acoustically excited flow through a circular orifice backed by a hexagonal cavity. Journal of Fluid Mechanics 693:367-401, doi:10.1017/jfm.2011.537

Zhang Q, Bodony DJ (2016) Numerical investigation of a honeycomb liner grazed by laminar and turbulent boundary layers. Journal of Fluid Mechanics 792:936-980, doi:10.1017/jfm.2016.79 